\title{
AKT Kinase Activity Is Required for Lithium to Modulate Mood-Related Behaviors in Mice
}

\author{
Jen Q Pan ${ }^{1,7}$, Michael C Lewis',7, Josh K Ketterman', Elizabeth L Clore', Misha Riley', Keenan R Richards', \\ Erin Berry-Scott', Xiulin Liu', Florence F Wagner', Edward B Holson', Rachael L Neve', Travis L Biechele ${ }^{3}$, \\ Randall T Moon ${ }^{3}$, Edward M Scolnick', Tracey L Petryshen*, ${ }^{*, 4,5}$ and Stephen J Haggarty*, , ,4,6 \\ IStanley Center for Psychiatric Research, Broad Institute of Harvard and MIT, 7 Cambridge Center, Cambridge, MA, USA; ${ }^{2}$ McGovern Institute for \\ Brain Research, Massachusetts Institute of Technology, Cambridge, MA, USA; ${ }^{3}$ Department of Pharmacology, Howard Hughes Medical Institute, \\ and Institute for Stem Cell and Regenerative Medicine, University of Washington School of Medicine, Seattle, WA, USA; ${ }^{4}$ Psychiatric and \\ Neurodevelopmental Genetics Unit and Molecular Neurogenetics Unit, Center for Human Genetic Research, Massachusetts General Hospital, \\ Boston, MA, USA; ${ }^{5}$ Department of Psychiatry, Harvard Medical School and Massachusetts General Hospital, Boston, MA, USA; ${ }^{6}$ Department of \\ Neurology, Haward Medical School and Massachusetts General Hospital, Boston, MA, USA
}

\begin{abstract}
Bipolar disorder (BP) is a debilitating psychiatric disorder, affecting 2\% of the worldwide population, for which the etiological basis, pathogenesis, and neurocircuitry remain poorly understood. Individuals with BP suffer from recurrent episodes of mania and depression, which are commonly treated with the mood stabilizer lithium. However, nearly half of BP patients do not respond adequately to lithium therapy and the clinically relevant mechanisms of lithium for mood stabilization remain elusive. Here, we modeled lithium responsiveness using cellular assays of glycogen synthase kinase 3 (GSK-3) signaling and mood-related behavioral assays in inbred strains of mice that differ in their response to lithium. We found that activating AKT through phosphosrylation of a key regulatory site (Thr308) was associated with lithium response-activation of signaling pathways downstream of GSK-3 in cells and attenuation of mood-related behaviors in mice - and this response was attenuated by selective and direct inhibition of AKT kinase activity. Conversely, the expression of constitutively active AKTI in both the cellular and behavioral assays conferred lithium sensitivity. In contrast, selective and direct GSK-3 inhibition by the ATP-competitive inhibitor CHIR9902I bypassed the requirement for AKT activation and modulated behavior in both lithium-responsive and non-responsive mouse strains. These results distinguish the mechanism of action of lithium from direct GSK-3 inhibition both in vivo and in vitro, and highlight the therapeutic potential for selective GSK-3 inhibitors in BP treatment. Neuropsychopharmacology (201 I) 36, I397-141 I; doi:10.1038/npp.201 I.24; published online 9 March 201।
\end{abstract}

Keywords: lithium; AKT; mania; depression; TCF/LEF; behavior

\section{INTRODUCTION}

Lithium was the first medication found to have moodstabilizing properties (Cade, 1949), and today remains a first-line treatment for bipolar disorder (BP). Despite the efficacy of lithium in preventing manic episodes and suicide (Bowden, 2000; Tondo and Baldessarini, 2009), half of BP patients do not respond to lithium therapy (Bowden, 2000; Geddes et al, 2010), and lithium treatment is associated with dose-limiting side effects (Le Roy et al, 2009).

*Correspondence: Dr SJ Haggarty and Dr TL Petryshen Stanley Center for Psychiatric Research, Broad Institute of Harvard and MIT, 7 Cambridge Center, Cambridge, MA 02142, USA,

Tel: + 617 7l4 7854,

E-mail: haggarty@chgr.mgh.harvard.edu (S) Haggarty) or petryshen@ chgr.mgh.harvard.edu (TL Petryshen)

${ }^{7}$ These authors contributed equally to this work.

Received 7 December 2010; revised 18 January 2011; accepted 27 January 2011
Therefore, there is a critical need to elucidate the molecular mechanism underlying lithium's modulation of affective behavior in order to understand the neurobiological basis of the observed variation in patient response.

Although many targets of lithium have been identified (Beaulieu et al, 2008, 2009; Berridge and Irvine, 1989; Klein and Melton, 1996), it remains unclear which of these are responsible for lithium's clinical efficacy. Lithium has been shown to inhibit glycogen synthase kinase (GSK)-3 kinase activity directly, via competition with magnesium, and indirectly, by increasing inhibitory phosphorylation of GSK-3 (Beaulieu et al, 2004, 2008; Chalecka-Franaszek and Chuang, 1999; De Sarno et al, 2002; Klein and Melton, 1996). Furthermore, GSK- $3 \alpha$ null or GSK-3 $\beta$ haploinsufficient mice phenocopy lithium's effect of attenuating aberrant behaviors (Beaulieu et al, 2004; Kaidanovich-Beilin et al, 2009; O'Brien et al, 2004). Conversely, mice overexpressing GSK-3 $\beta$ or carrying mutations preventing inhibitory phosphorylation of GSK-3 $\alpha$ (Ser21) and GSK-3 $\beta$ (Ser9) 
exhibit behaviors modeling psychiatric symptoms, as do mice with targeted disruption of AKT1, which phosphorylates and inactivates GSK-3 $\alpha$ (Ser21) and GSK-3 $\beta$ (Ser9) (Emamian et al, 2004; Lai et al, 2006; Polter et al, 2010; Prickaerts et al, 2006). AKT/GSK-3 signaling has also been implicated in the pathophysiology of neuropsychiatric disorders through biochemical and genetic association studies of patients (Emamian et al, 2004; Tan et al, 2008; Thiselton et al, 2008). In addition to lithium, antidepressants, antipsychotics, and other mood stabilizers also modulate GSK-3 activity (Beaulieu et al, 2009), further supporting its involvement in psychiatric illness. Various pharmacological probes of GSK-3 have been used to implicate GSK-3 kinase activity in the regulation of behavior in vivo (Beaulieu et al, 2007a; Gould et al, 2004). However, the specificity of these compounds has not been well characterized, and it is unclear whether the observed behavioral changes are due to GSK- $3 \beta$ inhibition or to non-selective effects on other kinases or targets.

As a key mediator between cortical input and basal ganglia output, the striatum has a critical role in dopaminemediated behavioral response to novelty and reward, whose disregulation underlies psychiatric disorders. Recent studies have implicated striatal AKT activation as a key regulator in dopamine-mediated hyperlocomotion in vivo. Reduced levels of phosphorylated AKT at a key regulatory site (p-AKT(308)) have been associated with hyperdopaminergic tone in dopamine transporter (DAT) knockout mice that display elevated spontaneous locomotion (Beaulieu et al, 2004; Zhuang et al, 2001). Conversely, increased striatal p-AKT was correlated with hypodopaminergic signaling in mice lacking dopamine D2 or D3 receptors (Beaulieu et al, 2007b), $\beta$-arrestin2 (Beaulieu et al, 2005), as well as in DAT overexpressing mice (Ghisi et al, 2009). In support for a key role of the striatum in mood neurocircuitry and the response to lithium, it was reported that lithium disrupts striatal $\beta$-arrestin 2/protein phosphatase 2A (PP2A)/AKT signaling complex ( $\beta$ Arr2:PP2A:AKT) and releases activated AKT, resulting in increased inhibitory phosphorylation of GSK-3 $\alpha \beta$ (Ser21/9) (Beaulieu et al, 2008). In this study, $\beta$ Arr2 knockout mice exhibited biochemical (p-AKT(308)) and behavioral (novelty-induced locomotion) insensitivity to lithium treatment compared with wild-type mice, presumably due to loss of the $\beta$ Arr2:PP2A:AKT complex. However, it remains unclear whether the kinase activity of AKT itself is necessary for the behavioral changes induced by lithium, or whether the effect of lithium treatment on AKT signaling in wild-type animals differs from the developmental and persistent perturbation of AKT activity derived from $\beta$ Arr2 deletion in knockout mice.

Here, we examined the role of AKT in lithium's mechanism of action in both cellular and mouse behavioral model systems. Using pharmacological loss-of-function studies and viral-mediated gain-of-function studies, we show that the kinase activity of AKT is required for lithium to regulate GSK-3 signaling in vitro, and that AKT activation in the striatum is critical for lithium to attenuate mood-related behavioral responses in vivo. In addition, we characterize a potent and selective GSK-3 inhibitor, CHIR99021, and demonstrate that it inhibits GSK-3 signaling in cells and attenuates mood-related behaviors in vivo independent of AKT activity. These results provide new insight into the molecular mechanisms through which lithium modulates mood-related behaviors in mice and validate pharmacological probes for dissecting the role of AKT/GSK-3 signaling in the pathogenesis of neuropsychiatric disorders.

\section{MATERIALS AND METHODS}

\section{Constructs and Antibodies}

Wild-type human AKT1 (9004), myristoylated-AKT1 with an N-terminal myristoylation sequence (9005), and PTEN (10750) constructs were purchased from Addgene. HSV-WT-AKT1, HSV-DN-AKT1 (containing a mutation in the catalytic site residue K179 to M179), HSV-CA-AKT1 (containing a N-terminal myristoylation sequence and a mutation of S473 to F473), and HSV- $\beta$-galactosidase viruses were prepared as described previously (Krishnan et al, 2008). Primary antibodies used were: mouse anti- $\beta$-arrestin2 (Santa Cruz, sc-13140), mouse immobilized anti-AKT (pan) antibody (Cell Signaling, 3653), mouse anti- $\beta$-actin (Sigma, A5441), rabbit anti-AKT (pan) (Cell Signaling, 4685), rabbit anti-AKT1 (Cell Signaling, 2938), rabbit antiphospho-AKT(308) (Cell Signaling, 2965), rabbit antiphospho-AKT(473) (Cell Signaling, 4060), rabbit antiGSK-3 $\alpha / \beta$ (Millipore, 05-903), rabbit anti-GSK-3 $\beta$ (Cell Signaling, 9315), rabbit anti-HA (Cell Signaling, 2367), and rabbit anti-phospho-GSK-3 $\beta$ (Ser9) (Cell Signaling, 9323). Anti-rabbit and anti-mouse secondary antibodies were purchased from GE Healthcare (NA931 and NA934) for western blot analysis. For immunohistochemistry, rabbit anti-AKT (Cell Signaling, 9272) and rabbit anti- $\beta$-galactosidase (Abcam, ab616) were used.

\section{Compounds}

LY294002, wortmannin, lithium chloride, sodium chloride, D-amphetamine sulfate, ketamine, xylazine, DMSO, and saline $(0.9 \% \mathrm{NaCl})$ were purchased from Sigma. CHIR99021 (Ring et al, 2003) and AKTI-17 (Inhibitor 17, Zhao et al, 2008) were synthesized according to published literature, and spectral properties were determined to be consistent with the proposed structures. For cellular studies, compounds were solubilized in $100 \%$ DMSO as $10 \mathrm{mM}$ stock solutions that were diluted accordingly. For behavioral studies, AKTI-17 was solubilized in 100\% DMSO and administered intracerebroventricularly in a $1.0 \mu \mathrm{l}$ volume at a rate of $0.5 \mu \mathrm{l} / \mathrm{min}$. D-amphetamine sulfate salt $(3.5 \mathrm{mg} / \mathrm{kg})$ and lithium chloride $(85 \mathrm{mg} / \mathrm{kg})$ were dissolved in saline and administered intraperitoneally in an injection volume of $5 \mathrm{ml} / \mathrm{kg}$. For intraperitoneal administration, CHIR99021 was dissolved in a solution consisting of $45 \%$ polyethylene glycol 400 (Fisher), 45\% saline, and 10\% DMSO and administered at doses of $3.1-25 \mathrm{mg} / \mathrm{kg}$ as indicated in an injection volume of $10 \mathrm{ml} / \mathrm{kg}$. For intracerebroventricular and intrastriatal administration, CHIR99021 was solubilized in $100 \%$ DMSO and administered in a $1.0 \mu \mathrm{l}$ volume at a rate of $0.5 \mu \mathrm{l} / \mathrm{min}$. 


\section{Cell Culture, Transfection, and Viral Infection}

The cell line HT-22 is a subclone of the HT-4 mouse hippocampal cell line (a gift from Dr Pamela Maher, Salk Institute). HEK293 (ATCC) and HT-22 cells stably expressing a firefly luciferase reporter gene driven by a promoter containing 12 copies of TCF/LEF-binding sequences and renilla luciferase driven by ubiquitous $\mathrm{EF} 1 \alpha$ promoter were constructed as previously described (Biechele and Moon, 2008), and maintained in DMEM supplemented with $10 \%$ heat-inactivated FBS (Invitrogen) and 1\% penicillin-streptomycin (Invitrogen). Reporter gene assays were conducted in antibiotic-free media. Transfection of plasmid DNA was performed in six-well plates ( $1 \mu \mathrm{g}$ DNA per well) using lipofectamine 1000 reagent (Invitrogen). At $24 \mathrm{~h}$ after transfection, cells were re-plated to 384-well plates for various treatments overnight $(16-20 \mathrm{~h})$, followed by reporter gene assays. For HSV infection, HT-22 cells were infected with HSV viruses $\left(1 \times 10^{4}\right.$ infection particles per $\mathrm{ml}$ ) and incubated at $37^{\circ} \mathrm{C}$. At $24 \mathrm{~h}$ after infection, cells were treated overnight with relevant compounds and assayed using DualGlo assay reagents (Promega).

\section{Immunoblot and Western Blot Analysis}

Cultured cells were collected in ice-cold lysis buffer containing $20 \mathrm{mM}$ Tris $(\mathrm{pH}=7.5), 150 \mathrm{mM} \mathrm{NaCl}, 1 \mathrm{mM}$ EDTA, and 1\% NP40 alternative supplemented with protease and phosphatase inhibitors (Roche Applied Science, 04693116001 and 04906837001$)$. The samples were incubated and gently rocked for $1 \mathrm{~h}$ before centrifuged at $12000 \times g$ for $10 \mathrm{~min}$. For tissue samples, neural tissues were dissected and collected in ice-cold PBS buffer, weighed, diced into small pieces, and homogenized by a mechanical homogenizer. The temperature for sample processing was maintained at $4{ }^{\circ} \mathrm{C}$ throughout all procedures. Samples were incubated on ice for $60 \mathrm{~min}$, transferred to microcentrifuge tubes, and centrifuged at $12000 \times g$ for $10 \mathrm{~min}$ at $4{ }^{\circ} \mathrm{C}$. The supernatant total cell lysate was subject to subsequent western blot analysis. Total cell lysates were boiled in $2 \times$ Laemmli sample buffer (Bio-Rad) before separation by SDS-PAGE gel (4-12\% gradient, Bio-Rad). Gels were transferred onto PVDF membrane (Millipore). Membranes were incubated overnight with primary antibodies in a solution of Tris-buffered saline containing Tween 20 (TBST, Sigma) and 5\% dry milk. Incubation of secondary antibodies was performed for $1 \mathrm{~h}$ at room temperature in a solution of $5 \%$ dry milk in TBST. Immunoblot staining was developed using Femto Peroxidase substrate (Thermo Scientific) and digitally captured using a molecular imaging station (Kodak) and analyzed using ImageJ software (National Institutes of Health).

\section{TCF/LEF Reporter Transcriptional Assay}

HEK293 and HT-22 reporter TCF/LEF reporter stable cell lines were seeded into 384-well culture plates (Corning, $3707)$ at 6000 and 3000 cells per well, respectively. At $24 \mathrm{~h}$ after plating, cells were treated overnight with relevant compounds and assayed using DualGlo assay reagents (Promega). Firefly luciferase intensity was normalized to renilla luciferase intensity, and TCF/LEF reporter activity was reported as the fold-change over the control treatment ( $\mathrm{NaCl}$, control transfection). Wnt-conditioned media is prepared from the L Wnt3a cell line (ATCC) based on the method described in Biechele et al (2009).

\section{Animals}

Male C57BL/6J and DBA/2J mice were purchased from the Jackson Laboratory (Bar Harbor, ME) at 10-weeks of age. Mice were housed four per cage on arrival (singly housed after surgery) in a room with a 12:12-h light-dark cycle and were allowed to acclimate for 1 week before behavioral procedures. Food and water were provided ad libitum. All procedures followed the National Institutes of Health Guide for the Care and Use of Laboratory Animals and were approved by the Massachusetts Institute of Technology Animal Care and Use Committee.

\section{Behavioral Procedures}

Amphetamine-induced hyperlocomotion (AIH) was examined in eight identical open-field chambers $(41.9 \times$ $40.6 \times 30.5 \mathrm{~cm}$; AccuScan Instruments). Activity was detected by infrared beam breaks and recorded automatically by VersaMax software (AccuScan Instruments). Distance traveled $(\mathrm{cm})$ data were automatically binned in 5-min intervals and total distance traveled post-amphetamine challenge was calculated for statistical analysis. On day 1, mice were habituated to the open field. Mice were administered a saline injection (to mock lithium or CHIR99021 injection on day 3), placed in an open field $40 \mathrm{~min}$ later for $20 \mathrm{~min}$ of activity monitoring, removed for saline injection (to mock D-amphetamine challenge on day 3 ), and subsequently placed back in the open field for an additional $30 \mathrm{~min}$. Mice utilized for AKT inhibitor experiments were also acclimated to the intracerebroventricular infusion procedure by gently restraining them while removing the dummy cannula, followed by $3 \mathrm{~min}$ of restraint, replacement of the dummy cannula and subsequent placement into the open field for activity monitoring. Day 2 was identical to day 1, with the exception that activity monitoring after mock $\mathrm{D}$-amphetamine injection lasted $40 \mathrm{~min}$ and AKTI-17 experiment mice received an intracerebroventricular saline infusion (2-min infusion followed by an additional minute for diffusion from the injection cannula) On day 3 , the D-amphetamine challenge day, mice utilized for lithium or CHIR99021 experiments were pretreated with $\mathrm{LiCl}(85 \mathrm{mg} / \mathrm{kg}$, i.p.), CHIR99021 $(3.1-25 \mathrm{mg} / \mathrm{kg}$, i.p.) or vehicle $40 \mathrm{~min}$ before placement in the open field, challenged 20 min later with D-amphetamine $(3.5 \mathrm{mg} / \mathrm{kg}$, i.p.), and subsequently monitored for an additional $80 \mathrm{~min}$. Mice utilized for AKT inhibitor experiments were infused with AKTI-17 $(10 \mu \mathrm{M}$ in $1 \mu \mathrm{l}$ total volume, i.c.v.) or DMSO vehicle ( $1 \mu \mathrm{l}$ total volume, i.c.v.) $45 \mathrm{~min}$ before $\mathrm{LiCl}$ or saline vehicle treatment, placed in the open field $40 \mathrm{~min}$ later for $20 \mathrm{~min}$ activity monitoring, followed by D-amphetamine challenge $(3.5 \mathrm{mg} / \mathrm{kg}$, i.p.) and an additional $80 \mathrm{~min}$ monitoring. The forced swim test (FST) was performed in five identical cylindrical chambers $(24 \times 15 \mathrm{~cm})$ containing warm water $\left(26 \pm 2{ }^{\circ} \mathrm{C}\right)$ at a depth of $12 \mathrm{~cm}$. For C57BL/6J mice, the FST protocol consisted of a single 6-min session, with behavior scored automatically 
(EthoVision; Noldus) for the final 4 min of the session. For DBA/2J mice, the FST protocol consisted of a 10-min session in which no data were recorded, followed the next day by a 6 -min session in which the last $4 \mathrm{~min}$ were scored (this was due to differences in baseline immobility times between C57BL/6J $(\sim 120 \mathrm{~s})$ and DBA/2J ( $\sim 40 \mathrm{~s})$ mice. Drugs were only administered before day 2 in the DBA/2J mice. Time spent immobile relative to vehicle controls were used as the dependent variable for all analyses.

\section{Statistical Analysis}

AIH data were analyzed using independent samples $t$-tests, one-way or two-way ANOVA of total distance travelled after amphetamine challenge, where appropriate. FST data were analyzed by two-way ANOVA. Post hoc analysis of results was analyzed using Tukey's HSD (Honestly Significant Difference) test. Post hoc statistical significance is indicated for all behavior results with $p<0.05$. All statistical analyses were performed with SPSS v. 18.0 (IBM). Cellular data were analyzed using one-way or twoway ANOVA, or $t$-test, where appropriate.

\section{Supplemental Methods}

Detailed methods on kinase activity, pharmacokinetics, histology, immunoprecipitation, and surgical procedures can be found in the supplementary material.

\section{RESULTS}

\section{AKT Kinase Activity Is Required for Lithium to Modulate Cellular GSK-3 Signaling}

In order to determine and dissect the possible molecular mechanisms that modulate lithium sensitivity, we sought to identity in vitro cellular models as surrogates for investigating signaling pathways and validating molecular probes for subsequent in vivo studies. Lithium is known to activate canonical Wnt signaling through inhibition of GSK-3 $\beta$, which in turn stabilizes $\beta$-catenin. The stabilized $\beta$-catenin then interacts with members of the T-cell factor (TCF)/ lymphoid enhancer-binding factor (LEF) family of transcription factors, and induces transcription both in vitro and in vivo (Hedgepeth et al, 1997; Klein and Melton, 1996; O'Brien et al, 2004). Therefore, we selected a TCF/LEFmediated transcriptional reporter assay to investigate the role of AKT signaling in lithium's mechanism of action (Klein and Melton, 1996; Yuan et al, 1999) in a variety of cell lines (SH-SY5Y, RKO, HEK293, and HT-22). These lines included HEK293 cells derived from human embryonic kidney, and HT-22 cells, an immortalized mouse hippocampal cell line sharing $>80 \%$ genetic similarity across a large panel of genomic sequence polymorphisms to the lithium-insensitive $\mathrm{FVB} / \mathrm{NJ}$ and SWR/J mouse strains (Supplementary Table 1; Gould et al 2007). Although both cell lines responded to Wnt3a-conditioned media, confirming the activity of the TCF/LEF reporter (data not shown), lithium treatment (overnight) induced robust TCF/LEF reporter activity in HEK293 cells but had no effect in HT22 cells at any dose tested (Figure 1a; Supplementary Figure $\mathrm{S} 1 \mathrm{~A})$. In addition, lithium did not activate an identical reporter gene with mutations in the TCF/LEF-binding site (data now shown), highlighting the specificity of the effect of lithium on TCF/LEF transcriptional activation in these cells. Furthermore, lithium treatment for $2 \mathrm{~h}$ increased TCF/ LEF-mediated transcription 1.7-fold over baseline; lithium treatment

for four hours increased TCF/LEF-mediated transcription $\sim 5$-fold over background; and overnight treatment of lithium increased TCF/LEF-mediated transcription $\sim 40$ fold from the baseline (data not shown). Western blot analysis was then performed to investigate whether the cellular response to lithium was correlated with AKT activation status. As shown in Figures $1 \mathrm{~b}$ and $c$, similar levels of total AKT1 were observed in HEK293 and HT-22 cells. While AKT was constitutively active and present in the active phosphorylated form in HEK293 cells, little AKT was phosphorylated in HT-22 cells. Lithium treatment for $2 \mathrm{~h}$ had no effect on p-AKT(308) in HT-22 cells, but caused a 30\% increase in p-AKT(308) levels in HEK293 cells (data not shown), consistent with previous reports in cultured cerebellar granule neurons (Chalecka-Franaszek and Chuang, 1999). Overnight treatment of lithium had no effect on pAKT(308) in either HT-22 cells or HEK293 cells (Figures $1 \mathrm{~b}$ and $\mathrm{c}$ ).

We next used the 'sensitive' HEK293 cellular model to examine whether reducing AKT activation could decrease lithium sensitivity (Figure 1d). Overexpression of exogenous PTEN, a lipid-phosphatase that reduces intracellular levels of $\mathrm{PIP}_{3}$ thereby negatively regulating AKT, attenuated $\sim 60 \%$ of the TCF/LEF activation by lithium (Figure 1e). Additionally, treating cells with LY294002 or wortmannin to inhibit PI3K, which normally increases intracellular PIP3 that positively regulates AKT, dose-dependently reduced TCF/LEF reporter activation by lithium (Figure 1f). Finally, to directly examine whether AKT activity is involved in lithium's modulation of GSK-3 signaling, we treated HEK293 cells with a highly selective, allosteric AKT inhibitor, AKTI-17 (Zhao et al, 2008, Inhibitor 17), which dose-dependently reduced the TCF/LEF reporter activation by lithium (Figure 1g). In contrast, AKTI-17 had no effect on TCF/LEF reporter activation in HEK293 cells stimulated with Wnt3a-conditioned media highlighting the differences between canonical Wnt signaling and lithium response (Supplementary Figure S1B). Taken together, these results establish that AKT kinase activity is required for lithium to modulate TCF/LEF-mediated transcription.

To further investigate whether lithium's modulation of TCF/LEF-mediated transcription depends specifically on the activation state of AKT, we tested whether an activated form of AKT was sufficient to invoke a lithium response in 'insensitive' HT-22 cells. Wild-type AKT1 (WT-AKT1) and constitutively active, myristoylated AKT1 (CA-AKT1) were transiently transfected into HT-22 cells and their expression was confirmed over the time course of the experiment (Supplementary Figure S2A). Overexpression of WT-AKT1 did not induce TCF/LEF reporter activation either in the presence or absence of lithium (Figure $1 \mathrm{~h}$ ). In contrast, overexpression of CA-AKT1, which is constitutively phosphorylated at both the Thr308 and Ser473 sites (Supplementary Figure S2B), induced a robust TCF/LEF reporter signal in the presence of lithium (Figure 1h; two-way ANOVA, $\mathrm{F}_{\text {treatment }}(1,128)=4639.7, p<0.00001$; 
a

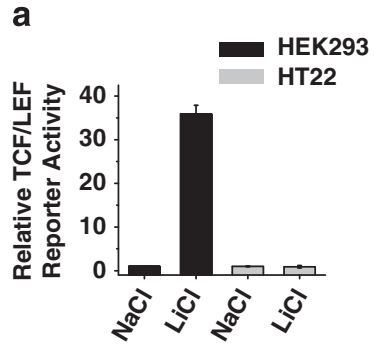

b

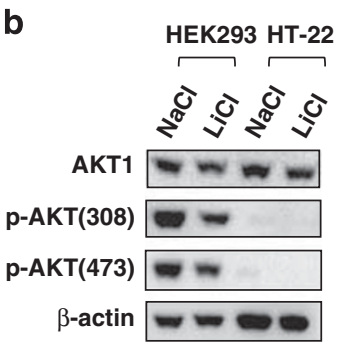

C

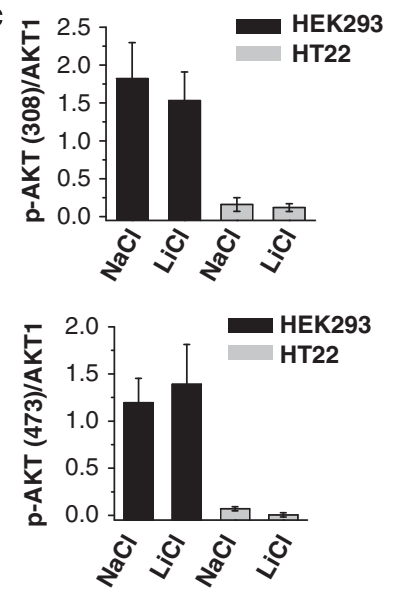

d
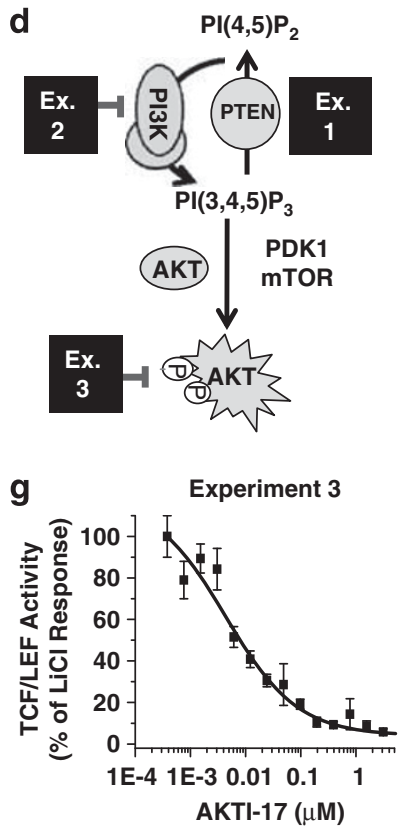

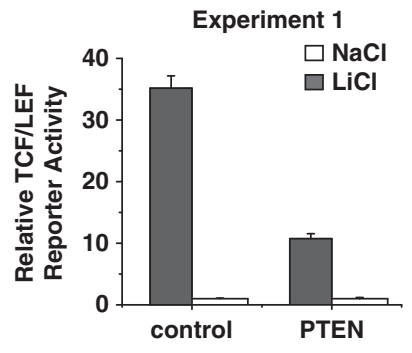

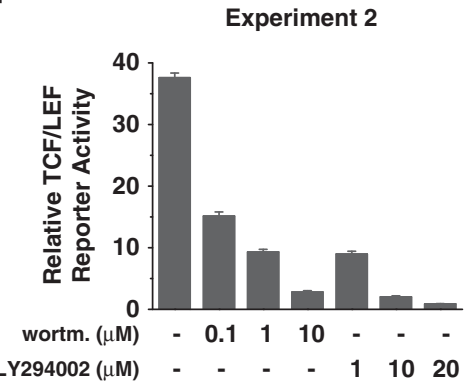
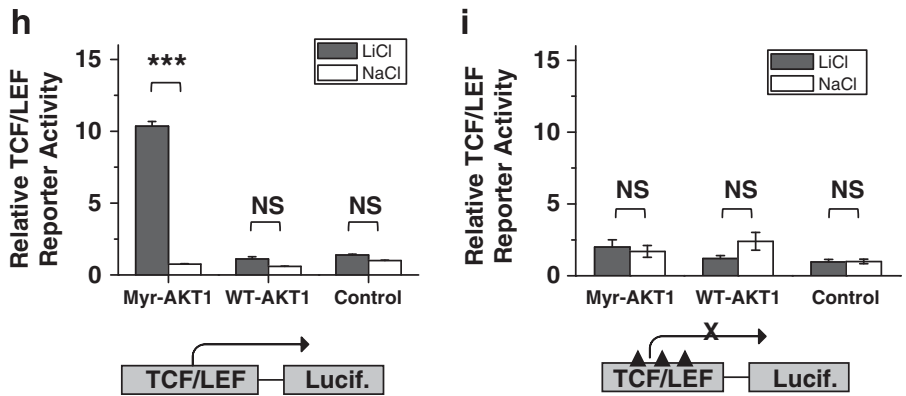

Figure I AKT kinase activity is required for the effect of lithium on TCF/LEF reporter activity. (a) HEK293 (black bars) and HT-22 (grey bars) expressing firefly luciferase driven by a TCF/LEF promoter were treated overnight with $\mathrm{LiCl}(10 \mathrm{mM})$, or $\mathrm{NaCl}(10 \mathrm{mM})$. TCF/LEF reporter activity was measured by Dual-Glo (Promega), and normalized to renilla luciferase activity under the control of ubiquitous EFI $\alpha$ promoter. TCF/LEF reporter activity is shown as the fold change relative to the control $\mathrm{NaCl}$ treatments. (b-c) Densitometric western blot analysis of phosphorylated and total AKTI expression in whole cell lysate from HEK293 and HT-22 cells treated overnight with $\mathrm{LiCl}(\mathrm{I} 0 \mathrm{mM})$ or $\mathrm{NaCl}$ ( $10 \mathrm{mM})$. $\beta$-Actin was used as the loading control for densitometry. Levels of p-AKT(308) and p-AKT(473) are shown as fold change relative to total AKTI within each treatment in HEK293 and HT-22 cells. For all results, data are mean \pm SEM from at least three independent experiments. (d) Schematic of PTEN, PI3K inhibitor, and AKTI- I7 interference with AKT signaling examined in three experiments (Ex.I, 2, 3). (e-h) TCF/LEF luciferase reporter assays of cells treated overnight with $\mathrm{LiCl}(\mathrm{I} / \mathrm{mM})$ or $\mathrm{NaCl}(\mathrm{IO} \mathrm{mM}$ ). Reporter activity was measured by Dual-Glo assay (Promega), and normalized to EFI $\alpha$ renilla luciferase activity. Data represent mean \pm SEM from at least three independent experiments. (e) HEK293 cells were transiently transfected with a plasmid expressing PTEN, or an empty plasmid (control), and treated with $\mathrm{LiCl}$ (dark grey bars) or $\mathrm{NaCl}$ (white bars). Relative TCF/LEF activity is shown as fold change relative to control (empty plasmid transfection and $\mathrm{NaCl}$ treatment). (f) HEK293 cells were treated with the PI3K inhibitors wortmannin (0. I, I, and I $0 \mu \mathrm{M}$ ) or LY294002 (I, I0, and 20 $\mu \mathrm{M})$ in the presence of LiCl. Relative TCF/LEF activity is shown as the fold change relative to NaCl treatment. (g) HEK293 cells were treated with AKTI- 17 (0.0003-3 $\mu$ M) overnight in the presence of $\mathrm{LiCl}$. The fraction of TCF/LEF reporter activity relative to the control (inhibitor absent) is plotted against the concentrations of AKTI-I7 $\left(\mathrm{IC}_{50}=0.008 \mu \mathrm{M}\right)$. (h) HT-22 cells were transfected with constitutively active myristoylated AKTI (CA-AKTI), wild-type AKTI (WT-AKTI), or reagents only (control), and treated with $\mathrm{LiCl}$ or $\mathrm{NaCl}$ overnight. TCF/LEF activity is shown relative to the control condition (control transfection and $\mathrm{NaCl}$ treatment). (i) HT-22 cells expressing firefly luciferase controlled by a mutated TCF/LEF promoter (mutation marked as black triangles) were transfected with Myr-AKTI, WT-AKTI, or reagents (control), and treated with $\mathrm{LiCl}$ or $\mathrm{NaCl}$ overnight. For all results, data are mean $\pm \mathrm{SEM}$. $* * * *<<0.00 \mathrm{I}$ compared to $\mathrm{NaCl}$ control.

$\mathrm{F}_{\text {transfection }}(2,128)=4630.3, p<0.00001 ; \mathrm{F}_{\text {interaction }}(2,128)=$ $4416.8, p<0.00001)$. Importantly, expression of CA-AKT1 in the presence of lithium did not activate an identical reporter gene with mutations in the TCF/LEF-binding site (Figure 1i; two-way ANOVA, $\mathrm{F}_{\text {treatment }}(1,240)=0.37$, $p=0.55 ; \mathrm{F}_{\text {transfection }}(2,240)=5.37, p=0.005 ; \mathrm{F}_{\text {interaction }}(2,240)$ $=1.97, p=0.14)$. This result indicates that the interaction between the activated form of AKT1 and lithium is not due to a general increase in transcriptional activation, further highlighting the specificity of these transcriptional effects of 
CA-AKT1 on GSK-3 signaling. Expression of CA-AKT1 in the absence of lithium had no effect on TCF/LEF-mediated transcription, indicating that while constitutive AKT1 can restore lithium sensitivity to HT-22 cells, the activity of CA-AKT1 alone is not sufficient to initiate TCF/LEFmediated transcription.

To further validate AKT's role in lithium response of HT-22 cells, and to validate a viral delivery system for expressing AKT variants in vivo in mouse brain, we repeated these HT-22 restoration studies with AKT1 delivered using herpes simplex virus (HSV). As observed in the transfection experiments above, expression of a constitutively active, myristoylated form of AKT1 (HSVCA-AKT1) stimulated TCF/LEF-mediated transcription in HT-22 cells in the presence of lithium, and had no effect when delivered without lithium treatment. In contrast, expression of wild-type AKT1 (HSV-WT-AKT1), dominantnegative AKT1 (HSV-DN-AKT1), or a control HSV vector expressing $\beta$-galactosidase (HSV- $\beta$-gal) had no effect (Supplementary Figure S2C; two-way ANOVA, $\mathrm{F}_{\text {treatment }}(1,144)=113.2, \quad p<0.0001 ; \mathrm{F}_{\text {virus groups }}(3,144)=$ $\left.87.3, p<0.0001 ; F_{\text {interaction }}(3,144)=84.8, p<0.0001\right)$. These results show that $\mathrm{AKT}$ activation is necessary and sufficient for lithium to induce TCF/LEF-mediated transcription.

\section{AKT Activation in the Striatum Correlates with the Ability of Lithium to Attenuate Mood-Related Behaviors in Inbred Mice}

A major limitation for the field of neuropsychiatric research is the lack of mouse models that faithfully recapitulate all aspects of human disease. Nonetheless, in order to assess the molecular mechanisms through which lithium alters the neurocircuitry involved in mood regulation and the role of $\mathrm{AKT}$ in this response, we examined the behavior of inbred mouse strains in models that are routinely used to assess the anti-manic and anti-depressant actions of candidate medications. We first considered the $\mathrm{AIH}$ paradigm that shows reasonable predictive validity for clinically prescribed mood stabilizers, including lithium administered either acutely or chronically (Arban et al, 2005; Dencker et al, 2008; Kim et al, 2008; Mavrikaki et al, 2010; O’Donnell and Gould, 2007; Perry et al, 2009). By inhibiting the dopamine and other monoamine transporters (Rothman and Baumann, 2006), amphetamine leads to motoric hyperactivity similar to that observed in BP patients in the manic phase (O'Donnell and Gould, 2007; Perry et al, 2009). Furthermore, similar to the variable efficacy of lithium treatment in BP patients, inbred mouse strains show differential sensitivity to lithium in attenuating the increased locomotion that occurs in response to amphetamine (Gould et al, 2007). Therefore, based on our cellular results above showing a key role for AKT in determining lithium response, we hypothesized that the observed behavioral changes in C57BL/6J and DBA/2J mice in the AIH paradigm (Gould et al, 2007), which have previously been characterized as lithium-responsive and lithium non-responsive, respectively, would correlate with levels of AKT activation.

Since Beaulieu et al $(2004,2008)$ demonstrated that lithium treatment increases striatal levels of phosphorylated AKT (p-AKT(308)), we first examined p-AKT(308) abundance in striatal tissue from mice pretreated with lithium $(85 \mathrm{mg} / \mathrm{kg}$, i.p.) $1 \mathrm{~h}$ before $\mathrm{D}$-amphetamine administration $(3.5 \mathrm{mg} / \mathrm{kg}$, i.p.). Lithium-responsive C57BL/6J mice treated with lithium chloride alone, or with lithium chloride and D-amphetamine, showed significantly higher levels of p-AKT(308), 30 min after D-amphetamine administration than animals treated with vehicle or D-amphetamine alone (Figures $2 \mathrm{a}$ and $\mathrm{b}$, black bars, one-way ANOVA, $\mathrm{F}(3,48)=8.1, p=0.008)$. In contrast, lithium-insensitive DBA/2J mice showed no significant change in p-AKT(308) level with any treatment (Figures $2 \mathrm{a}$ and $\mathrm{b}$, grey bars, $\mathrm{F}(3,48)=0.88, p=0.49$, one-way ANOVA). Furthermore, increased p-AKT(308) levels in C57BL/6J striatum returned to basal levels $2 \mathrm{~h}$ after lithium treatment in the absence or presence of amphetamine, indicating that the striatal activation of AKT by lithium in C57BL/6J is time dependent (Supplementary Figure S3A).

To further clarify the biochemical effect induced by lithium, we next examined striatal GSK- $3 \beta$ phosphorylation in both strains of mice treated with lithium (i.p., $85 \mathrm{mg} / \mathrm{kg}$ ) compared with vehicle treatment. Although the total levels of AKT1 or GSK-3 $\beta$ in either mouse strain remained unaltered by lithium treatment (Figure 2c), the level of striatal p-GSK-3 $\beta$ (9) increased significantly in C57BL/6J treated with lithium, paralleling the increase in striatal p-ATK(308) (Figures $2 \mathrm{~d}$ and e two-tail paired $t$-test: for pAKT, $t(18)=-5.0, p=0.038$; for $\mathrm{p}-\mathrm{GSK}-3 \beta t(18)=-4.7$, $p=0.04)$. In contrast, lithium treatment alone did not induce changes in the levels of p-GSK-3 $\beta(9)$ or p-ATK(308) in DBA2/J striatum (Figures $2 \mathrm{~d}$ and e, two-tail paired $t$-test: pAKT: $t(18)=0.25, \quad p=0.25 ; \quad \mathrm{p}-\mathrm{GSK}-3 \beta: \quad t(18)=-0.69$, $p=0.55)$. To determine if the failure of lithium to increase striatal p-AKT(308) level was specific to the DBA/2J strain, three other strains reported to be lithium insensitive (FVB/ $\mathrm{NJ}, \mathrm{C} 3 \mathrm{H} / \mathrm{HeJ}, 129 \mathrm{~S} 6 / \mathrm{SvEv}$ ) were examined (Gould et al, 2007). Similar to DBA/2J mice, p-AKT(308) levels in striatal tissue did not increase after lithium administration in any of these lithium-insensitive mouse strains (Supplementary Figure S4).

We next determined that, while both C57BL/6J and DBA/2J strains displayed similar levels of basal locomotion (data not shown) and hyperlocomotion induced by D-amphetamine (3.5 mg/kg, i.p.), lithium treatment $(85 \mathrm{mg} / \mathrm{kg}$, i.p.) significantly attenuated hyperlocomotion by $40 \%$ in C57BL/6J mice compared with vehicle treatment, but had no effect in DBA/2J mice (Figure 2f, two-way ANOVA, $\mathrm{F}_{\text {treatment }}(1,32)=6.24, p=0.018 ; \mathrm{F}_{\text {strain }}(1,32)=1.41$, $\left.p=0.410 ; \mathrm{F}_{\text {interaction }}(1,32)=5.10, p=0.030\right)$. These results show a correlation between AKT activation in the striatum and lithium responsiveness in the $\mathrm{AIH}$ behavioral model. Taken together with our findings on p-AKT(308) levels, these findings support the notion that inhibitory Ser9 phosphorylation of GSK-3 $\beta$ as a result of lithium's activation of AKT may be a key determinant of lithium response in vivo.

Recently, Beaulieu et al have demonstrated that mice lacking a complex of $\beta$ Arr2:PP2A:AKT in the striatum because of genetic deletion of $\beta$ Arr 2 exhibit behavioral and biochemical insensitivity to lithium treatment. To determine the similarity between these findings and our results with inbred strains, we sought to determine whether a difference in striatal $\beta$ Arr2:PP2A:AKT complex formation 
a

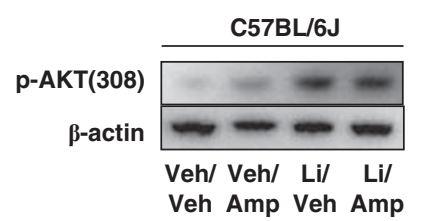

DBA/2J

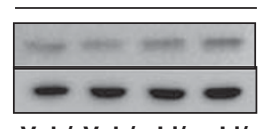

Veh/ Veh/ Li/ Li/
(1)

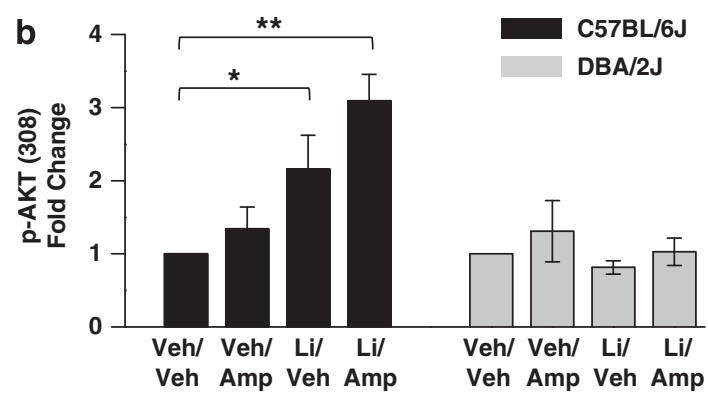

C

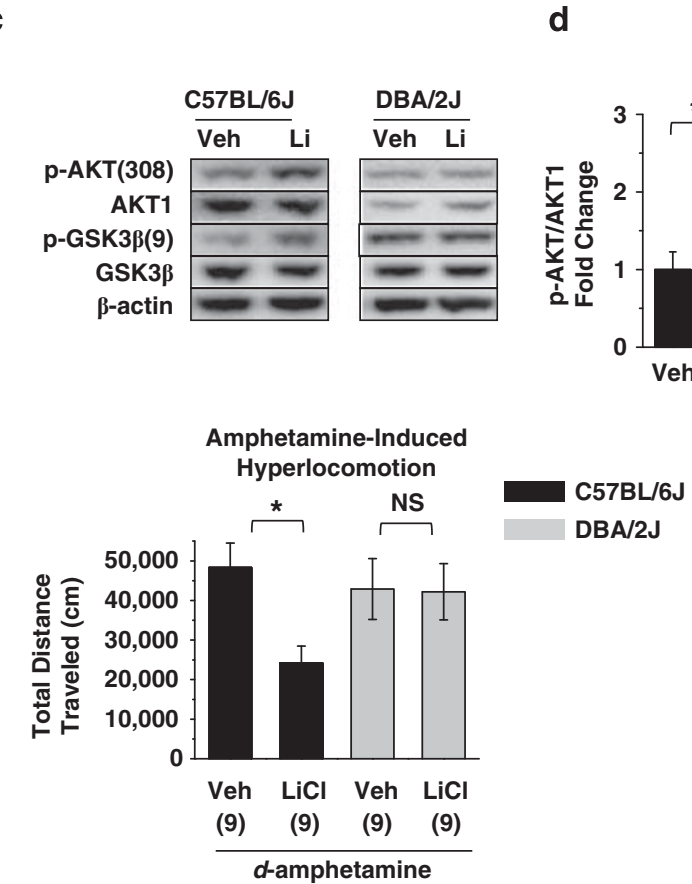

C57BL/6J DBA/2J

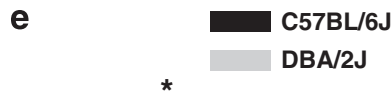

f g

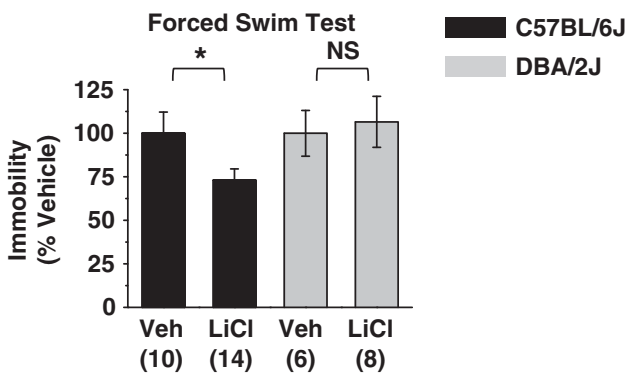

Figure 2 Lithium induces distinct functional responses in vivo in inbred mouse strains that vary in AKT activation. (a, b) Densitometric western blot analysis of relative expression of p-AKT(308) in total cell lysate from striatum of C57BL/6J (black) and DBA/2J (grey) mice that were injected with saline vehicle or $\mathrm{LiCl}(85 \mathrm{mg} / \mathrm{kg}$, i.p.) $60 \mathrm{~min}$ before saline vehicle or D-amphetamine $(3.5 \mathrm{mg} / \mathrm{kg}$, i.p.) challenge. Striatal tissues were taken $30 \mathrm{~min}$ after D-amphetamine (or vehicle) administration. Expression of p-AKT (308) was normalized to a $\beta$-actin loading control, and fold change in p-AKT(308) compared with vehicle treatment in each mouse strain. Data represent the average of three independent western blot analyses for each animal and four animals in each treatment group $(n=12)$. (c-e) Densitometric western blot analysis of relative expression of p-AKT(308) and p-GSK-3 $\beta(9)$ in striatal lysate of C57BL/6J (black) and DBA/2J (grey) mice injected with saline vehicle or LiCl $(85 \mathrm{mg} / \mathrm{kg}$, i.p.) $90 \mathrm{~min}$ before tissue harvest. Expression of p-AKT(308) and p-GSK-3 $\beta(9)$ was normalized to the level of total AKTI or GSK-3 $\beta$ in each sample, and fold change in phosphorylated protein compared with vehicle treatment in each mouse strain is quantified. Data represent the average of three independent western blot analyses for each animal and three animals in each treatment group. (f) DBA/2J or C57BL/6J mice were injected with saline vehicle or LiCl (85 mg/kg, i.p.) $60 \mathrm{~min}$ before D-amphetamine (3.5 mg/ $/ \mathrm{kg}$, i.p.) challenge. Total distance traveled $(\mathrm{cm})$ over $80 \mathrm{~min}$ after D-amphetamine injection was quantified. Number of animals per group $(\mathrm{n})$ is indicated. (g) DBA/2] or C57BL/6] mice were treated with saline vehicle or $\mathrm{LiCl}(85 \mathrm{mg} / \mathrm{kg}$, i.p.) $45 \mathrm{~min}$ before the FST. Time spent immobile (\%) is relative to the vehicle group immobility time. Number of animals per group $(n)$ is indicated. For all results, data are mean \pm SEM. $* p<0.05$ and $* * p<0.01$ compared with vehicle-treated mice; NS, $p>0.05$.

and stability in the presence of lithium could explain the lithium insensitivity observed in DBA/2J mice. We found that stable $\beta$ Arr2:AKT and PP2A:AKT complexes could be isolated from the striatum of both C57BL/6J and DBA/2J mice in the absence of lithium (Supplementary Figures S3C-E). In C57BL/6J mice, addition of lithium (1 and $10 \mathrm{mM}$ ) to the striatal lysate resulted in a significant disruption of both $\beta$ Arr2:AKT and PP2A:AKT complexes. In DBA/2J mice, a high concentration of lithium $(10 \mathrm{mM})$ was also able to disrupt both complexes, whereas a lower concentration (1 mM) disrupted $\beta$ Arr2:AKT but not PP2A:AKT complexes (Supplementary Figures S3C-E). These results show that stable striatal Arr2:AKT complexes exist equally in both strains of mice, and there is no apparent difference in the stability of Arr2:AKT in the presence of 1 or $10 \mathrm{mM}$ lithium. We interpret these data to suggest that differences in the disruption of the Arr2:AKT complex are unlikely to account for the observed biochemical deficits in striatal pAKT activation or behavioral insensitivity to lithium in DBA/2J mice. However, because PP2A is a highly ubiquitous cellular phosphatase and the PP2A:AKT complex may have many cellular roles, we cannot rule out a role for PP2A:AKT in altering the Arr2:AKT ternary complex in other inbred strains of mice, other brain regions, or at different doses of lithium.

To extend these studies to an additional behavioral paradigm relevant to mood disorders that involves other brain circuits and neurotransmitter systems than those involved in $\mathrm{AIH}$, we next examined the behavior of C57BL/ $6 \mathrm{~J}$ and DBA/2J mouse strains in the FST. The FST is 
proposed as a model of behavioral despair, thought to be mediated in part by serotonergic and noradrenergic transmission, in which both acute and chronic lithium treatment alters the behavior similar to clinical antidepressants (O'Donnell and Gould, 2007). We found that acute lithium ( $85 \mathrm{mg} / \mathrm{kg}$, i.p.) resulted in an anti-depressantlike effect, as determined by reduced immobility time, in C57BL/6J mice compared with vehicle treatment. However, acute lithium did not have an anti-depressantlike effect in DBA/2J mice as compared with vehicle treatment (Figure $2 \mathrm{~g}$; two-way ANOVA $\mathrm{F}_{\text {treatment }}(1,34)=$ $5.01, \quad \mathrm{p}=0.032, \quad \mathrm{~F}_{\text {strain }}(1,34)=2.96, \quad p=0.094 ; \quad \mathrm{F}_{\text {interaction }}$ $(1,34)=0.99, \quad p=0.325)$. These findings validate the C57BL/6J strain as lithium-responsive and the DBA/2J strain as lithium non-responsive in two behavioral models relevant to mood disorders.

\section{Inhibition of AKT Activity Blocks Lithium's Modulation} of Mood-Related Behaviors in C57BL/6J Mice

Having determined through both loss- and gain-of-function experiments that the kinase activity of AKT is required for lithium response in cellular assays of GSK-3 signaling, we next utilized AKTI-17 to examine whether AKT kinase activity is also a key component of the behavioral response to lithium. Using C57BL/6J mice, direct brain administration of AKTI-17 alone (0.1, 1 and $10 \mu \mathrm{M}$ i.c.v.) did not alter basal (data not shown) or D-amphetamine-induced locomotion (Figure 3a), suggesting AKT kinase activity is not required for the increased motor response to amphetamine. In contrast, AKTI-17 (10 $\mu \mathrm{M}$ i.c.v.) blocked lithium's attenuation of hyperlocomotion induced by amphetamine (Figure $3 \mathrm{~b}$ and $\mathrm{c}$; one-way ANOVA, $\mathrm{F}_{\text {treatment }}(2,26)=5.12$, $p=0.013)$. Post hoc analysis revealed significantly reduced hyperlocomotion after lithium treatment $(p=0.027$, compared with vehicle control), and reversal of this attenuation by AKTI-17 treatment $(p=0.640$ compared with vehicle control). These data demonstrate that, on AKT inhibition,
C57BL/6J mice no longer respond to lithium, indicating that AKT activation is required for lithium to modulate behavior.

\section{Constitutively Active AKT Restores Lithium Sensitivity in DBA/2J Mice}

Since the expression of HSV-CA-AKT1 was sufficient to restore lithium sensitivity in cells (Supplementary Figure S2C), we examined whether the same HSV-CAAKT1 viral construct could restore the behavioral response to lithium. Using DBA/2J mice, HSV-CA-AKT1, HSV-WT$\mathrm{AKT} 1$, or HSV- $\beta$-gal viruses were infused bilaterally into the striatum, and successful AKT1 overexpression was validated by immunohistochemistry (Supplementary Figure S5A, B). Before $\mathrm{D}$-amphetamine challenge, there were no differences in basal locomotor activity between the three groups, indicating that the viral delivery did not affect motor performance (Supplementary Figure S5C). On D-amphetamine treatment $(3.5 \mathrm{mg} / \mathrm{kg}$, i.p.), however, lithium significantly attenuated hyperlocomotion in mice expressing HSV-CA-AKT1 compared with vehicle control (Figure 4c; two-tail paired $t$-test, $t(17)=3.19, p=0.005)$. In contrast, lithium had no effect in mice expressing either HSV-WTAKT1, (Figure $4 \mathrm{~d} ; t(15)=1.02, p=0.32$ ) or the HSV- $\beta$-gal control, compared with vehicle treatment (Figure 4b; $t(13)=0.18, p=0.86)$. These results show that expression of activated AKT1 in striatum effectively restored lithium's ability to attenuate the amphetamine response in otherwise lithium-insensitive DBA/2J mice. These findings are consistent with the cellular assay of GSK-3 signaling in which expression of activated AKT was sufficient to restore lithium-sensitivity to otherwise insensitive HT-22 cells (Figure 1g).

Direct GSK-3 Inhibition by an ATP-Competitive

Inhibitor is Effective in Cellular and Mouse Behavioral Models of Lithium Insensitivity

Having established a key role for AKT activation in lithium response, we sought to clarify whether GSK- $3 \alpha$ and GSK-3 $\beta$, a

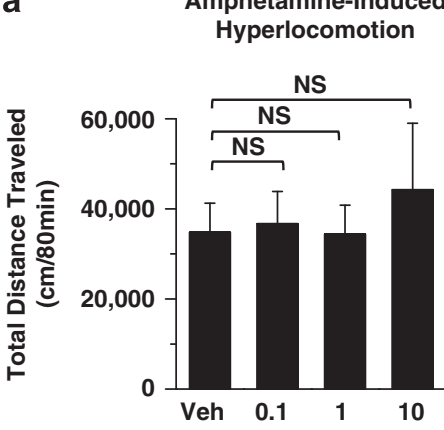

$\begin{array}{llll}(8) & \text { (8) } & \text { (8) } & \text { (9) }\end{array}$

AKTl-17 $(\mu \mathrm{M})$

d-amphetamine $(3.5 \mathrm{mg} / \mathrm{kg})$

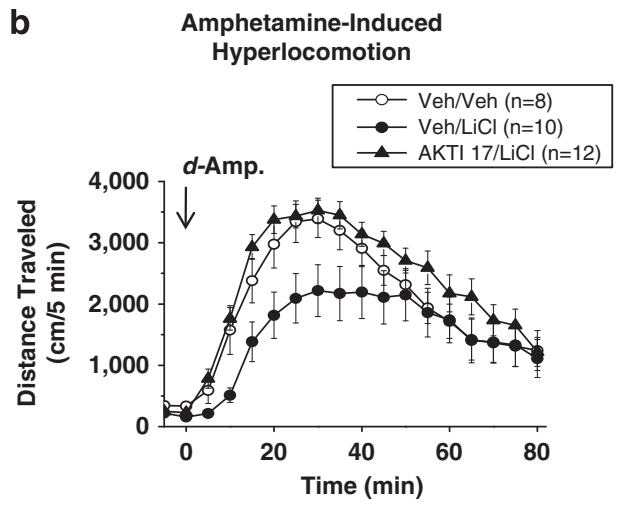

Amphetamine-Induce
Hyperlocomotion

$\because-\operatorname{Veh} / \operatorname{Veh}(\mathrm{n}=8)$ Veh/LiCl $(n=10)$
C Amphetamine-Induced Hyperlocomotion

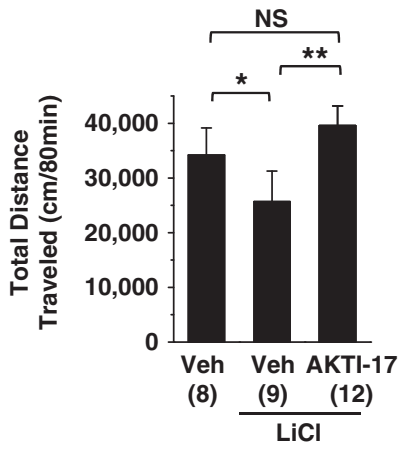

Figure 3 AKT kinase activity is required for lithium to attenuate AlH in C57BL/6) mice. (a) C57BL/6) mice were pretreated with AKTI- 17 (0.I, I or I0 $\mu \mathrm{M}$ in 100\% DMSO, i.c.v.) or 100\% DMSO vehicle (Veh) $60 \mathrm{~min}$ before D-amphetamine challenge ( $3.5 \mathrm{mg} / \mathrm{kg}$, i.p.). Total distance traveled was quantified for $80 \mathrm{~min}$ after D-amphetamine injection. (b, c) C57BL/6) mice were pretreated with AKTI- 17 (I0 $\mu \mathrm{M}$ in 100\% DMSO, i.c.v.) or I00\% DMSO vehicle (Veh) $45 \mathrm{~min}$ before administration of lithium ( $85 \mathrm{mg} / \mathrm{kg}$, i.p.) or saline vehicle $(V e h)$, which occurred $60 \mathrm{~min}$ before D-amphetamine challenge (time 0; $3.5 \mathrm{mg} / \mathrm{kg}$, i.p.). Distance $(\mathrm{cm})$ traveled within 5 -min bins is plotted against the total 80 min recording time $(\mathrm{b})$, and total distance $(\mathrm{cm})$ traveled was quantified in the 80-min period after D-amphetamine challenge (c). Number of animals per group ( $n$ ) is indicated. For all results, data are mean \pm SEM. $* p<0.05$ and *** $p<0.01$ compared with vehicle-treated mice; NS, $p>0.05$. 
a
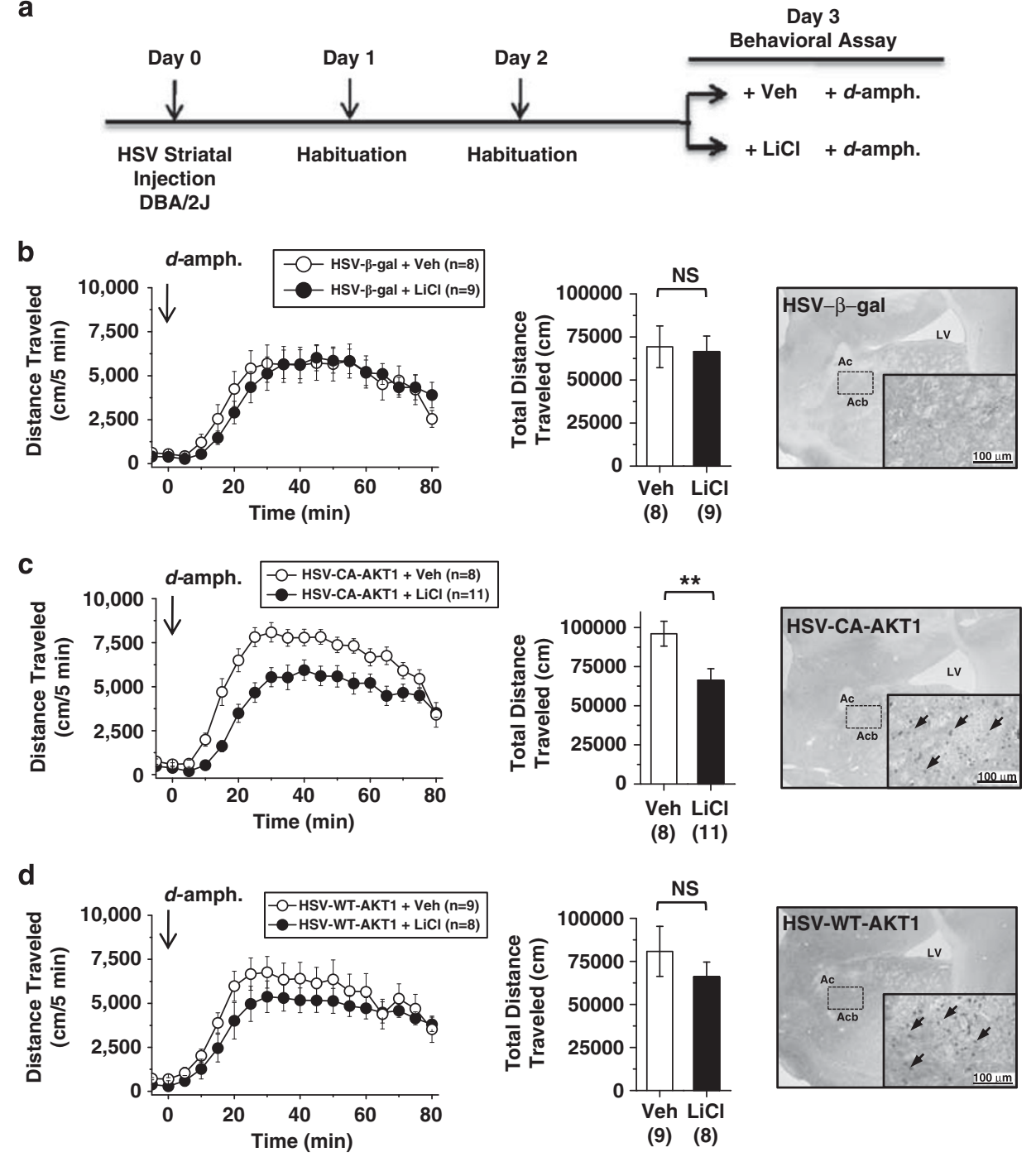

Figure 4 Active AKT restores lithium sensitivity to DBA/2] mice in the AlH paradigm. (a) Schematic of viral-mediated delivery of AKT forms and testing of lithium sensitivity of DBA/2 I mice in the AlH assay. (b-d) DBA/2 I mice were striatally injected with HSV viruses $\left(\sim \mid \times 10^{8}\right.$ infection particles per ml) expressing (b) $\beta$-galactosidase (HSV- $\beta$-gal), (c) a constitutively active form of AKT (HSV-CA-AKTI), or (d) wild-type AKT (HSV-WT-AKTI) 3 days before behavioral testing to allow expression of the delivered genes. On the test day, mice were administrated lithium ( $85 \mathrm{mg} / \mathrm{kg}$, i.p.) or saline vehicle (Veh) $60 \mathrm{~min}$ before D-amphetamine challenge (time $0 ; 3.5 \mathrm{mg} / \mathrm{kg}$, i.p.), and activity was recorded for an additional $80 \mathrm{~min}$. Distance (cm) traveled is shown for each 5 -min bin across the total recording time (left panels), and for the 80-min period after D-amphetamine challenge (right panels). Representative histology sections $(4 \times$ and $20 \times)$ are shown for each viral delivery. Insets, arrows point to AKT-immunoreactivity. Quantification of AKT-immunoreactive neurons is shown in Supplementary Figure S5A. Representative histology sections $(4 \times$ and $20 \times)$ for HSV- $\beta$-gal expression are shown in Supplementary Figure S5B. Data shown are mean \pm SEM. $* * * p<0.005$, as compared with vehicle control mice. Number of animals per group ( $n$ ) is indicated. Ac, anterior commissure, Acb, nucleus accumbens, Lv, lateral ventricle.

known downstream targets of AKT, are involved in the behavioral and cellular effects of lithium. To identify a potent and selective small-molecule inhibitor of GSK- $3 \alpha / \beta$ that would ultimately be suitable to probe GSK-3 function in vivo, we profiled the binding affinity of several known GSK-3 inhibitors against a panel of 359 recombinant kinases using KINOMEscan technology (Fabian et al, 2005). Consistent with previous reports that examined a much more restricted set of 80 kinases (Bain et al, 2007), the aminopyrimidine CHIR99021 and the thiazole urea AR-A014418 were found to be highly selective toward GSK- $3 \alpha / \beta$. In contrast, the commonly used inhibitors BIO $\left(\left(2^{\prime} Z, 3^{\prime} E\right)\right.$-6-bromoindirubin-3'-oxime $)$ and SB-216763 targeted numerous other kinases, limiting their utility as probes of GSK-3 function in vivo (Figure 5a). We subsequently confirmed that CHIR99021 was a potent inhibitor of GSK-3 $\beta$ ( $\mathrm{IC}_{50}=4 \mathrm{nM}$ ) in enzymatic assays measuring GSK-3 $\beta$ activity, and it was active in the cellular $\mathrm{TCF} / \mathrm{LEF}$ reporter assay $\left(\mathrm{EC}_{50}=1.5 \mu \mathrm{M}\right)$ (Figure $5 \mathrm{~b}$ ). Furthermore, CHIR99021 induced robust TCF/LEF reporter activation in both lithium-sensitive HEK293 and lithiuminsensitive HT-22 cells (Figure $5 \mathrm{c}$ ). These results indicate that direct GSK-3 inhibition is sufficient to drive TCF/LEFmediated transcription independent of the activation state of AKT, thereby bypassing a key component of lithium's mechanism of action.

As administration of AR-A014418 was ineffective in the AIH assay in C57BL/6J mice (Supplementary Figure S6A), 
a

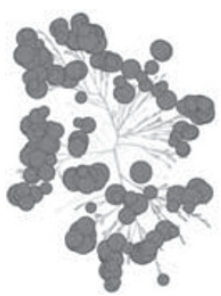

BIO

b

Functional Properties

\begin{tabular}{rcc}
\hline & $\begin{array}{l}\text { GSK-3 } \beta \\
\mathrm{IC}_{50}(\mathrm{nM})\end{array}$ & $\begin{array}{c}\mathrm{TCF}^{\mathrm{T} / L E F} \\
\mathrm{EC}_{50}(\mu \mathrm{M})\end{array}$ \\
\hline BIO & 8 & 7 \\
SB-216763 & 12 & 17 \\
AR-A014418 & 142 & $>25$ \\
CHIR99021 & 4 & 1.5 \\
\hline
\end{tabular}

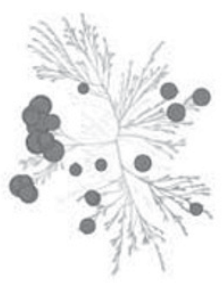

SB-216763

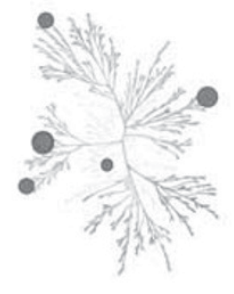

AR-A014418

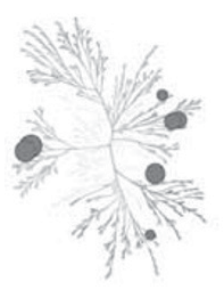

CHIR99021

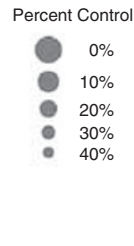

AR
C

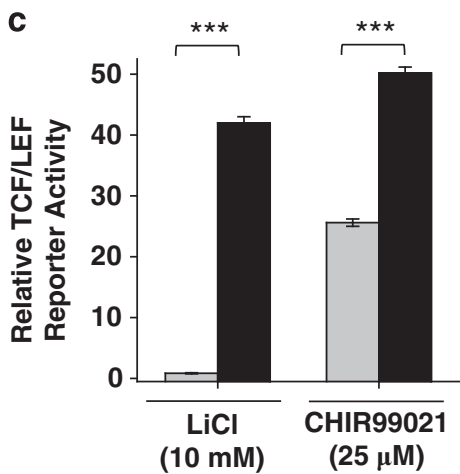

$\square \mathrm{HT}-22$

HEK293
5 CHIR9902 I is a highly selective inhibitor of GSK-3. (a) Profiles of BIO, SB-216763, AR-A0I44I8, and CHIR9902I using KINOMEscan profiling technology (Ambit). Compounds were screened against 359 kinases for competitive binding with active site binders. Competitive binding relative to positive controls (\% of control) is shown, with larger circles indicate higher-affinity binding. The complete list of kinases screened, as well as competitive binding data (\% control), are available in Supplementary Table 2. (b) Summary of functional properties of CHIR9902I. GSK-3 $\beta$ enzymatic activity determined by KinaseGlo assay (Promega) and concentrations of each compound that produce 50\% inhibition $\left(\mathrm{IC}_{50}\right)$ are shown. TCF/LEF reporter activity was measured in HEK293 cells and concentrations of each compound that produce $50 \%$ activation (EC 50 ) are shown. (c) Activity of CHIR9902I in the TCF/LEF reporter assay in HEK293 and HT-22 cells. TCF/LEF luciferase reporter activity was measured by Dual-Glo assay (Promega), and normalized to EF I $\alpha$ renilla luciferase activity. Data represent mean \pm SEM from at least three independent experiments. $* * * * p<0.001$. and is known to have poor brain penetration (Vasdev et al, 2005), we selected CHIR99021 to evaluate the role of GSK-3 in mood-related behaviors. We first analyzed CHIR99021 brain tissue pharmacokinetic properties after systemic administration to both $\mathrm{C} 57 \mathrm{BL} / 6 \mathrm{~J}$ and $\mathrm{DBA} / 2 \mathrm{~J}$ mice. CHIR99021 (12.5 mg/kg, i.p.) resulted in a maximum brain concentration $\left(C_{\max }\right)$ of $160 \mathrm{nM}$ in both strains $60 \mathrm{~min}$ after administration (Figure 6a). Thus, subsequent behavioral tests at this dose of CHIR99021 would be expected to surpass the brain concentrations required to bind GSK- $3 \alpha / \beta$ $\left(K_{\mathrm{d}}=17\right.$ and $5.7 \mathrm{nM}$ respectively, data not shown), with minimal binding to other kinases based on the selectivity profiling described above (Supplementary Table 2). Consistent with these pharmacokinetic data, and the previous genetic and pharmacological data supporting a role for GSK-3 in regulation of the locomotor response to amphetamine, we found that acute, systemic CHIR99021 administration $(12.5 \mathrm{mg} / \mathrm{kg}$, i.p., as well as lower doses, see Supplementary Figure S6B), significantly decreased hyperlocomotion in both mouse strains (two-way ANOVA $\mathrm{F}_{\text {treatment }}(1,18)=41.21, \quad p=0.001 ; \quad \mathrm{F}_{\text {strain }}(1,18)=15.60$, $\left.p=0.001 ; F_{\text {interaction }}(1,18)=8.11, p=0.011\right)$, with a $\sim 30 \%$ reduction in $\mathrm{C} 57 \mathrm{BL} / 6 \mathrm{~J}$ and $\mathrm{a} \sim 60 \%$ reduction in $\mathrm{DBA} / 2 \mathrm{~J}$ (Figure 6b). Importantly, there were no differences in motor activity after CHIR99021 administration in the absence of amphetamine, suggesting that nonspecific motor effects of the compound were not responsible for the attenuation observed in the AIH paradigm (see Supplementary Figure S6E). To confirm that the observed attenuation of hyperlocomotion by systemic CHIR99021 administration was not due to effects on peripheral tissues, and to gain insight into the brain circuits underlying GSK-3's role in the locomotor response to D-amphetamine, CHIR99021 was administered either i.c.v or intrastriatally $(0.3$ or $3.0 \mu \mathrm{M}$ in $1 \mu$ total volume) to $\mathrm{C} 57 \mathrm{BL} / 6 \mathrm{~J}$ mice, which reduced $\mathrm{AIH}$ by $\sim 30$ and $80 \%$, respectively (Supplementary Figures S6C and S6D). Taken together with our results showing restoration of lithium sensitivity by viral-mediated expression of activated AKT in the striatum, these data indicate that AKT/GSK-3 signaling in striatal circuits is a critical regulator of mood-related behaviors in mice.

We next extended these studies by examining whether CHIR99021 administration could modulate other moodrelated behavioral models in addition to the AIH paradigm. We tested CHIR99021 (25 mg/kg, i.p.) in the FST model and observed $\sim 30 \%$ decreased immobility in both lithium-sensitive $\mathrm{C} 57 \mathrm{BL} / 6 \mathrm{~J}$ mice and lithium-insensitive $\mathrm{DBA} / 2 \mathrm{~J}$ mice (Figure $6 \mathrm{c}, \mathrm{F}_{\text {treatment }}(1,32)=20.81, p=0.001$, $\mathrm{F}_{\text {strain }}(1,32)=0.71, \quad p=0.41 ; \quad \mathrm{F}_{\text {interaction }}(1,32)=2.05$, $p=0.16$, two-way ANOVA). These data provide evidence that selective and potent GSK-3 inhibition through acute and systemic administration of CHIR99021 has antidepressant-like properties in both lithium-sensitive and -insensitive mouse strains, in agreement with our findings in the AIH model.

Finally, to determine whether the cellular and behavioral effects of inhibiting GSK-3 by CHIR99021 were independent of AKT activity, we evaluated the impact of blocking AKT kinase activity on the efficacy of CHIR99021 in both the cellular reporter assay and mouse behavioral models. 
In Vivo Pharmacokinetic Properties

\begin{tabular}{ccc}
\hline CHIR99021 & Plasma & Brain \\
\hline $\mathrm{C}_{\max }(\mu \mathrm{M})$ & 15.09 & 0.16 \\
$\mathrm{~T}_{1 / 2}(\mathrm{hr})$ & 0.87 & 0.46 \\
AUC $\left(\mu \mathrm{M} / \mathbf{k g}^{\star} \mathrm{hr}\right)$ & 10.93 & 0.15 \\
\hline
\end{tabular}

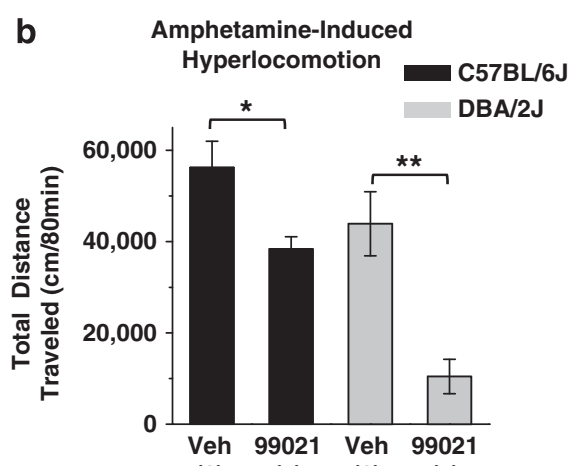

d

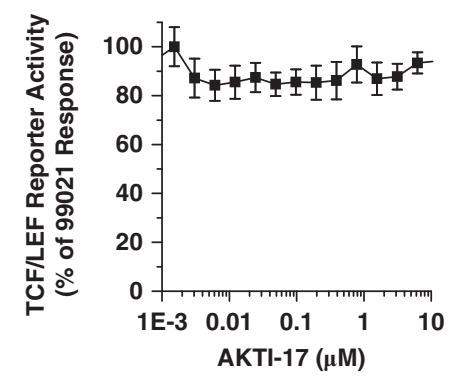

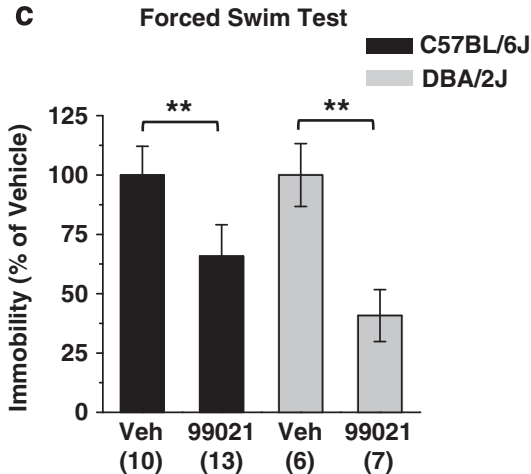

e Amphetamine-Induced Hyperlocomotion (C57BL/6J mice)

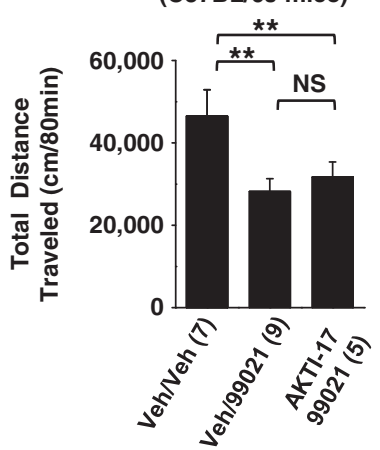

Figure 6 CHIR9902I attenuates mood-related behaviors in lithium-responsive and non-responsive mouse strains. (a) Selected pharmacokinetic properties of CHIR9902 I systemic administration (I $2.5 \mathrm{mg} / \mathrm{kg}$, i.p) in C57BL/6J plasma and brain tissue. AUC (area under the curve from the time of dosing to the time of the last observation), $T_{1 / 2}$ (terminal half-life) and $C_{\max }$ (maximum concentration) were determined one hour after CHIR9902I administration. The full pharmacokinetic report of CHIR9902I is available on request. For all results, data are mean \pm SEM. * $p<0.05$. Number of animals per group ( $n$ ) is indicated. (b) C57BL/6J (black bars) and DBA/2J (grey bars) mice were treated with vehicle (45\% PEG400, 45\% saline, I0\% DMSO) or CHIR9902I ( $12.5 \mathrm{mg} / \mathrm{kg}$, i.p.) $60 \mathrm{~min}$ before D-amphetamine challenge (time 0; $3.5 \mathrm{mg} / \mathrm{kg}$, i.p.), and the total distance (cm) traveled in the following 80 min was quantified. (c) C57BL/6J (black bars) and DBA/2J (grey bars) mice were treated with vehicle (45\% PEG400, 45\% saline, I0\% DMSO) or CHIR9902I (25 mg/kg, i.p.) $45 \mathrm{~min}$ before the FST. Time spent immobile is shown as the percentage relative to the vehicle group. (d) HEK293 cells were treated with AKTI- I7 (0.0 I-

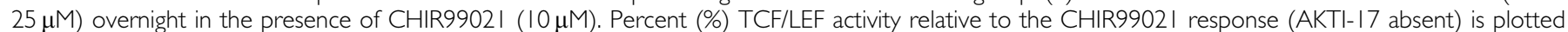
against the concentrations of AKTI- I7. (e) C57BL/6I mice were pretreated with AKTI-I7 (I0 $\mu \mathrm{M}$ in I00\% DMSO, i.c.V.) or I00\% DMSO vehicle (Veh) $45 \mathrm{~min}$ before administration of CHIR9902I (I2.5 mg/kg, i.p.) or vehicle (45\% PEG400, 45\% saline, I0\% DMSO) $60 \mathrm{~min}$ before D-amphetamine administration (time 0; $3.5 \mathrm{mg} / \mathrm{kg}$, i.p.). Total distance traveled $(\mathrm{cm}$ ) was quantified for $80 \mathrm{~min}$ after D-amphetamine challenge. Post hoc comparison, $p=0.003$ (CHIR9902I vs Veh), $p=0.57$ (CHIR9902I vs AKTI- 17). For all results, data are mean \pm SEM. $* p<0.05$ and $* * p<0.01$ compared with vehicle-treated mice; NS, $p>0.05$.

AKTI-17 treatment did not reduce the TCF/LEF reporter activity induced by CHIR99021 $(10 \mu \mathrm{M})$ in HEK293 cells (Figure 6d). Furthermore, pretreatment of mice with AKTI$17(10 \mu \mathrm{M}$ in $1 \mu \mathrm{l}$ total volume; i.c.v. $)$ did not interfere with AIH attenuation by CHIR99021 (12.5 mg/kg, i.p.) in C57BL/6J mice (Figure 6e, one-way ANOVA, $\mathrm{F}_{\text {treatment }}(2,18)=6.04$, $p=0.01)$. These data contrast with those described above in which AKT inhibition blocked the lithium response in HEK293 cells (Figure 1g) and in C57BL/6J mice (Figures 3b and c), highlighting the differences between the effects of lithium and direct GSK-3 inhibition. Overall, these results show that ATP-competitive inhibition of GSK-3 by CHIR99021 has a functional effect both in vitro and in vivo independent of AKT activation, clearly distinguishing the mechanism of lithium action from that of direct GSK-3 inhibition.

\section{DISCUSSION}

We report here that AKT activation determines lithium response in cellular assays of GSK-3 signaling and in two mouse behavioral assays that are relevant to neuropsychiatric disease (Beaulieu et al, 2004, 2008; Chalecka-Franaszek and Chuang, 1999; De Sarno et al, 2002). We found that inhibition of AKT by AKTI-17 blocked the behavioral response to lithium in C57BL/6 J mice in the AIH model, demonstrating that AKT kinase activity is critical for 
lithium's efficacy in this behavioral assay (Figure 3). Moreover, striatal expression of constitutively active AKT1 induced a behavioral response in the AIH model with lithium treatment in normally lithium-insensitive DBA/2J mice, while expression of wild-type AKT1 did not (Figure 4). Notably, activation of AKT alone was not sufficient to alter AIH behavior in DBA/2J mice, as shown by the lack of effect of constitutively active AKT1 in the absence of lithium (Figure 4). While previous reports support the view that lithium indirectly inhibits GSK-3 via AKT activation in vivo, this study is the first to conclusively demonstrate using pharmacological probes and viral-mediated gene delivery that the kinase activity of AKT within the striatum, as compared with correlated changes in the phosphorylation status of AKT and its presence within a complex with $\beta$ Arr2 and PP2A, is required for lithium to modulate behavior in mice. Moreover, the short-term nature of our manipulations of AKT kinase activity negates the possibility of potential confounding effects of chronic AKT changes, as seen in previous reports of knockout mice (Beaulieu et al, 2005). Our data support the notion that in contrast to a direct, ATP-competitive GSK-3 inhibitor, CHIR99021, which caused behavioral changes regardless of AKT activation state, the direct inhibition of GSK-3 by acute single dose lithium treatment is insufficient to exert behavioral effects when AKT activity is reduced with the pharmacological agent AKTI-17, or by natural genetic variation in the lithium non-responsive DBA/2J mice. We cannot rule out the possibility that a direct effect of lithium on GSK-3 kinase activity does not occur in vivo. Alternatively, AKT activation may modulate additional, yet to be discovered, signaling pathways involved in mood-related behaviors. Gaining insight into this question will benefit from further analysis using genetically modified strains of mice with alterations in AKT isoforms and the use of additional pharmacological probes targeting pathways and molecules affected by AKT and GSK-3 signaling.

Defining the kinetics of AKT activation/inhibition by dopamine and other monoamine neurotransmitters may shed light on how AKT regulation modulates behavior. Dopamine signaling driven by amphetamine or cocaine administration has been reported to induce a biphasic change in the phosphorylation state of AKT within striatum, with an initial increase in the level of pAKT(308) within $15 \mathrm{~min}$ followed by a decrease in pAKT after $60 \mathrm{~min}$ (Beaulieu et al, 2004; Brami-Cherrier et al, 2002; McGinty et al, 2008). Conversely, the dopamine receptor antagonist haloperidol induces an increase in striatal pAKT within $2 \mathrm{~h}$ (Emamian et al, 2004). Our data reveal that acute inhibition of AKT in the striatum through the administration of the selective AKT inhibitor, AKTI-17, does not disrupt spontaneous activity or hyperlocomotion induced by amphetamine, demonstrating that modulating AKT activity alone is not able to induce changes in neurotransmission regulating locomotion. This interpretation is further corroborated by results showing that mice lacking AKT1 exhibit normal spontaneous activity as well as hyperactivity induced by exploration, but have impaired working memory under neurochemical challenge (Lai et al, 2006). These findings suggest AKT1 is not necessary for locomotion but is required for higher-level prefrontal function. Furthermore, our results showed that constitutively activated AKT, when virally delivered into the striatum of $\mathrm{DBA} / 2 \mathrm{~J}$ mice, did not provoke changes in spontaneous activity, although a trend (not statistically significant) toward elevated activity in response to amphetamine was noted. This finding suggests that acute, direct striatal AKT activation does not dampen dopamine-mediated locomotion as would be predicted based on data from the AKT1 knockout mice. Taken together, our results indicate that AKT activation or inhibition has minimal effect on dopamine-mediated locomotion in the absence or presence of amphetamine; however, AKT activation within the striatum is necessary for lithium to be efficacious in the AIH paradigm.

We demonstrated that administration of the selective, potent, ATP-competitive GSK-3 inhibitor CHIR99021 was well-tolerated and significantly ameliorated mood-related behaviors in mice. The behavioral effects of CHIR99021 are likely due to its specific inhibition of GSK-3, because it is highly selective for GSK- $3 \alpha / \beta$ over 359 other kinases at the concentration achieved in the mouse brain (Figure $5 \mathrm{a}$; Supplementary Table 2). While several other GSK-3 inhibitors have been examined previously in mood-related models in rodents, the selectivity and pharmacokinetic properties of these inhibitors have largely not been addressed, making it unclear whether the behavioral effects were truly due to inhibition of GSK-3 or another target of the inhibitors. Furthermore, the thiazole urea AR-A014418 has been reported to attenuate both spontaneous and amphetamine-induced activity in rats (Gould et al, 2004). We saw no effect in the AIH paradigm, however, when the same dose of AR-A014418 was administered systemically to C57BL/6J mice (Supplementary Figure S6A). This apparent discrepancy could be due to species-specific effects (mouse $v s$ rat), different methodology (novel $v s$ habituated environment) or amphetamine dose $(0.5 v s 3.5 \mathrm{mg} / \mathrm{kg})$ in the rat study compared with ours, or other factors. Thus, to our knowledge, our study is the first to provide a detailed characterization of biochemical, cellular, and pharmacokinetic properties of a highly specific GSK-3 inhibitor that shows activity in mood-related behavioral models in mice. Since acute systemic administration of CHIR99021 regulates the neurocircuitry involved in mood-related behaviors, our results validated CHIR99021 as a valuable probe for future studies aiming to dissect the signaling pathways through which GSK-3 activity modulates behavior. Given that inhibition of AKT kinase activity by AKTI-17 did not disrupt CHIR99021's ability to modulated AIH or TCF/LEF-mediated transcriptional activity (Figures $6 \mathrm{~d}$ and e), and DBA/2J mice lacking lithium-induced AKT activation responded to CHIR99021 in the AIH and FST behavioral models (Figures $6 \mathrm{~b}$ and $\mathrm{c}$ ), our data clearly demonstrate that direct GSK-3 inhibition bypasses the need for active AKT in vitro and in vivo.

In agreement with our CHIR99021 behavioral findings, previous transgenic mouse studies have shown that heterozygous mice with a deletion of one of the two copies of GSK$3 \beta$ display reduced activity in response to amphetamine and, conversely, overexpressing GSK-3 $\beta$ caused hyperlocomotion (Beaulieu et al, 2004; Polter et al, 2010; Prickaerts et al, 2006). Furthermore, a recent study reported behaviors modeling aspects of manic and depressive symptoms in knockin mice expressing GSK-3 $\alpha / \beta$ that are resistant to Ser21/9-mediated inhibition (Polter et al, 2010). The 
severity of the depressive-like phenotype in these mice was negatively correlated with the level of p-AKT(308), consistent with an important role for AKT in modulating mood-related behaviors. Interestingly, chronic lithium administration still partially attenuated the amphetamine response in these mice in the absence of inhibitory phosphorylation of GSK- $3 \alpha / \beta$. Further investigation of the role of AKT in lithium's modulatory effects in these mice may clarify whether other AKT substrates underlie lithium's behavioral effect in these mice in the absence of Ser21/9mediated inhibition of GSK-3 $\alpha / \beta$.

In contrast to our findings with the AIH paradigm, viralmediated expression of CA-AKT in the striatum was not able to restore lithium sensitivity in the FST in DBA2/J mice (data not shown). This highlights the regional specificity of activated AKT in dopamine-mediated behaviors (eg, AIH) in the presence of lithium, as FST is thought to be mediated in part by serotonergic and noradrenergic transmission likely through brain regions distinct from striatum. Investigating AKT-GSK-3 interaction in other brain areas, including the prefrontal cortex and hippocampus, could reveal region-specific functions of $\mathrm{AKT}$, and may also explain some of the differences between the current study using selective and acute manipulation of striatal AKTGSK-3 compared other mutant mouse studies with more global alterations of this pathway. Despite the fact that direct inhibition of GSK-3 with CHIR99021 results in an attenuation of AIH behavior, we found that short-term manipulation of AKT kinase activity directly with AKT-I17 or overexpression of constitutively active AKT1 in the striatum had no effect on basal locomotion or on the ability of D-amphetamine to induce hyperlocomotion. This latter difference suggests that AKT signaling may contribute to maintaining the proper tone of GSK-3 activity, but it is not the sole determinant of GSK-3 signaling that is relevant to the AIH paradigm. Consistent with this conclusion, GSK-3 is known to integrate signaling input from multiple pathways, including other kinases, such as PKA, p70S6K, PKC and phosphatases, such as PP2A and PP1 (Beaulieu et al, 2009).

As we used a TCF/LEF reporter in a cellular assay of GSK$3 \beta$-mediated signaling, we have begun to investigate whether the $\beta$-catenin/TCF signaling pathway mediates lithium's anti-hyperactivity effect in vivo. To date, we have not obtained convincing data that either changes in $\beta$-catenin levels or its phosphorylation state in brain correlates with the observed changes in behavior under acute conditions. These findings differ from what has been reported with chronic (eg, 21 day) lithium treatment, which has been shown to increase $\beta$-catenin levels in brain lysate or cytosolic extracts of cortex, prefrontal cortex, hypothalamus, and hippocampus by (Böer et al, 2008) and others. However, Klein et al detected only a subtle ( $\sim 30 \%)$ increase in $\beta$-catenin levels in hypothalamus, and no significant change in frontal cortex, hippocampus, or cerebellum (O'Brien et al, 2004), suggesting that detection of altered $\beta$-catenin levels may be highly dependent on experimental conditions. Nonetheless, it is possible that the TCF/LEFmediated reporter assay provides only a surrogate assay for measuring GSK-3 pathway modulation and GSK-3 inhibition in vivo. Therefore, it remains unclear which substrates downstream of GSK-3 are affected independently by lithium's activation of AKT and are the relevant neural substrates affecting behavior under acute treatment.

As for the molecular basis for the requirement of activated AKT to modulate mood-related behaviors, a possible model is that, since lithium binds competitively with magnesium, which is required for the phosphotransferase activity of GSK-3, and activated AKT is a negative regulator of GSK-3 through inhibitory Ser9 phosphorylation that creates a pseudo-substrate inhibitor from the $\mathrm{N}$-terminal tail of GSK-3, the two modes of inhibition together may be needed to sufficiently dampen otherwise constitutively active GSK-3 activity to a threshold level that must be reached before sustained cellular and behavioral effects occur. An alternative model is one in which phosphorylated, active AKT and lithium function through a yet-to-be-defined signaling pathway paralleling GSK-3 inhibition that is necessary for lithium to modulate cellular and behavioral function. Future experiments examining lithium-sensitive and -insensitive mouse strains, lithium in combination with GSK-3 inhibitors, and manipulating other lithium targets such as inositol monophosphatases (IMPases) in the presence of GSK-3 inhibitors, will help determine whether lithium's effect in cellular and behavioral models requires meeting a threshold of GSK-3 inhibition or operates through pathways parallel to, or upstream of, GSK-3 inhibition.

Recent studies have reported abnormal regulation of inhibitory GSK-3 serine phosphorylation in fresh peripheral blood mononuclear cells from medication-free BP patients (Polter et al, 2010). Although our findings of parallel AKT activation and lithium response are based on acute gain- and loss-of-function manipulations that do not appropriately model the chronic signaling changes expected in patients, it is tempting to speculate that AKT activation in peripheral tissues may predict efficacy of lithium treatment, and that investigation of AKT and GSK-3 activity in these tissues may clarify the molecular mechanisms underlying lithium responsiveness in BP patients.

Interestingly, lithium is not effective in treating psychosis in schizophrenia patients (Leucht et al, 2004) and, associated with this lack of response, a previous study reported reduced AKT1 and phospho-AKT1 levels in postmortem brain samples and lymphoblastoid cell lines from schizophrenia patients (Emamian et al, 2004). Given our findings that functional AKT kinase activity is necessary for the mood-stabilizing effect of lithium on mouse behavior, the data are consistent with a loss of AKT activation in schizophrenia patients. Additional studies in animal models of psychosis may help elucidate the role of AKT signaling in schizophrenia.

In summary, our results from in vitro cellular signaling models and in vivo mood-related behavioral paradigms provide compelling evidence for a model in which AKT activation is required for lithium's mechanism of action. In contrast, highly specific GSK-3 inhibition by CHIR99021 regulated mouse behavior in an AKT-independent manner. These findings highlight the therapeutic potential for selective GSK-3 inhibitors in BP treatment (Beaulieu et al, 2009; Gould et al, 2006; O'Brien and Klein, 2009), particularly for those patients who do not respond to or tolerate lithium treatment. A more complete understanding of the role of AKT/GSK-3 in the action of lithium and other 
mood stabilizers may provide insight into the abnormal neural processes underlying BP and facilitate the development of new pharmacotherapies for the treatment of BP and potentially other psychiatric diseases.

\section{ACKNOWLEDGEMENTS}

We thank Drs Li-Huei Tsai, Daniel Fass, Jon Madison, Pamela Sklar, Melanie Leussis, and Roy Perlis for critical reading of the paper. We thank Dr Jennifer Moran for technical assistance with HT-22 cell genotype analysis. We thank Dr. Michael Moyer for assistance with the AKT inhibitor experiments. We thank Drs M Mazei-Robison and E Nestler for providing HSV-AKT1 constructs. This work was supported by grants from the National Institutes of Health (RL1GM084437 to EMS, administratively linked to RL1CA133834, RL1HG004671, and UL1RR024924; MH087442-01 to JP and SJH) and the Stanley Medical Research Institute (to EMS, SJH, TLP). RTM is an investigator of the HHMI.

\section{DISCLOSURE}

The authors declare no conflict of interest.

\section{REFERENCES}

Arban R, Maraia G, Brackenborough K, Winyard L, Wilson A, Gerrard P et al (2005). Evaluation of the effects of lamotrigine, valproate and carbamazepine in a rodent model of mania. Behav Brain Res 158: 123-132.

Bain J, Plater L, Elliott M, Shpiro N, Hastie CJ, McLauchlan H et al (2007). The selectivity of protein kinase inhibitors: a further update. Biochem J 408: 297-315.

Beaulieu JM, Gainetdinov RR, Caron MG (2007a). The Akt-GSK-3 signaling cascade in the actions of dopamine. Trends Pharmacol Sci 28: 166-172.

Beaulieu JM, Gainetdinov RR, Caron MG (2009). Akt/GSK3 signaling in the action of psychotropic drugs. Annu Rev Pharmacol Toxicol 49: 327-347.

Beaulieu JM, Marion S, Rodriguiz RM, Medvedev IO, Sotnikova TD, Ghisi V et al (2008). A beta-arrestin 2 signaling complex mediates lithium action on behavior. Cell 132: 125-136.

Beaulieu JM, Sotnikova TD, Marion S, Lefkowitz RJ, Gainetdinov RR, Caron MG (2005). An Akt/beta-arrestin 2/PP2A signaling complex mediates dopaminergic neurotransmission and behavior. Cell 122: 261-273.

Beaulieu JM, Sotnikova TD, Yao WD, Kockeritz L, Woodgett JR, Gainetdinov RR et al (2004). Lithium antagonizes dopaminedependent behaviors mediated by an AKT/glycogen synthase kinase 3 signaling cascade. Proc Natl Acad Sci USA 101: 5099-5104.

Beaulieu JM, Tirotta E, Sotnikova TD, Masri B, Salahpour A, Gainetdinov RR et al (2007b). Regulation of Akt signaling by D2 and D3 dopamine receptors in vivo. J Neurosci 27: 881-885.

Berridge MJ, Irvine RF (1989). Inositol phosphates and cell signalling. Nature 341: 197-205.

Biechele TL, Moon RT (2008). Assaying beta-catenin/TCF transcription with beta-catenin/TCF transcription-based reporter constructs. Methods Mol Bio 468: 99-110.

Biechele TL, Adams AM, Moon RT (2009). Transcription-based reporters of Wnt/beta catnein signaling. Cold Spring Harb Protoc 2009: pdb.prot5223.
Böer U, Cierny I, Krause D, Heinrich A, Lin H, Mayr G et al (2008). Chronic lithium salt treatment reduces CRE/CREB-directed gene transcription and reverses its upregulation by chronic psychosocial stress in transgenic reporter gene mice. Neuropsychopharmacology 33: 2407-2415.

Bowden CL (2000). Efficacy of lithium in mania and maintenance therapy of bipolar disorder. J Clin Psychiatry 61(Suppl 9): $35-40$.

Brami-Cherrier K, Valjent E, Garcia M, Pages C, Hipskind RA, Caboche J (2002). Dopamine induces a PI3-kinase-independent activation of Akt in striatal neurons: a new route to cAMP response element-binding protein phosphorylation. J Neurosci 22: 8911-8921.

Cade JF (1949). Lithium salts in the treatment of psychotic excitement. Med J Aust 2: 349-352.

Chalecka-Franaszek E, Chuang DM (1999). Lithium activates the serine/threonine kinase Akt-1 and suppresses glutamate-induced inhibition of Akt-1 activity in neurons. Proc Natl Acad Sci USA 96: 8745-8750.

De Sarno P, Li X, Jope RS (2002). Regulation of Akt and glycogen synthase kinase-3 beta phosphorylation by sodium valproate and lithium. Neuropharmacology 43: 1158-1164.

Dencker D, Dias R, Pedersen ML, Husum H (2008). Effect of the new antiepileptic drug retigabine in a rodent model of mania. Epilepsy Behav 12: 49-53.

Emamian ES, Hall D, Birnbaum MJ, Karayiorgou M, Gogos JA (2004). Convergent evidence for impaired AKT1-GSK3beta signaling in schizophrenia. Nat Genet 36: 131-137.

Fabian MA, Biggs III WH, Treiber DK, Atteridge CE, Azimioara MD, Benedetti MG et al (2005). A small molecule-kinase interaction map for clinical kinase inhibitors. Nat Biotechnol 23: 329-336.

Geddes JR, Goodwin GM, Rendell J, Azorin JM, Cipriani A, Ostacher MJ et al (2010). Lithium plus valproate combination therapy versus monotherapy for relapse prevention in bipolar I disorder (BALANCE): a randomised open-label trial. Lancet 375: 385-395.

Ghisi V, Ramsey AJ, Masri B, Gainetdinov RR, Caron MG, Salahpour A (2009). Reduced D2-mediated signaling activity and trans-synaptic upregulation of D1 and D2 dopamine receptors in mice overexpressing the dopamine transporter. Cell Signal 21: 87-94.

Gould TD, Einat H, Bhat R, Manji HK (2004). AR-A014418, a selective GSK-3 inhibitor, produces antidepressant-like effects in the forced swim test. Int J Neuropsychopharmacol 7: 387-390.

Gould TD, Picchini AM, Einat H, Manji HK (2006). Targeting glycogen synthase kinase- 3 in the CNS: implications for the development of new treatments for mood disorders. Curr Drug Targets 7: 1399-1409.

Gould TD, O’Donnell KC, Picchini AM, Manji HK (2007). Strain differences in lithium attenuation of d-amphetamine-induced hyperlocomotion: a mouse model for the genetics of clinical response to lithium. Neuropsychopharmacology 32: 1321-1333.

Hedgepeth CM, Conrad LJ, Zhang J, Huang HC, Lee VM, Klein PS (1997). Activation of the Wnt signaling pathway: a molecular mechanism for lithium action. Dev Biol 185: 82-91.

Kaidanovich-Beilin O, Lipina TV, Takao K, van Eede M, Hattori S, Laliberte $\mathrm{C}$ et al (2009). Abnormalities in brain structure and behavior in GSK-3alpha mutant mice. Mol Brain 2: 35 .

Kim WY, Kim S, Kim JH (2008). Chronic microinjection of valproic acid into the nucleus accumbens attenuates amphetamine-induced locomotor activity. Neurosci Lett 432: 54-57.

Klein PS, Melton DA (1996). A molecular mechanism for the effect of lithium on development. Proc Natl Acad Sci USA 93: 8455-8459.

Krishnan V, Han MH, Mazei-Robison M, Iniguez SD, Ables JL, Vialou $\mathrm{V}$ et al (2008). AKT signaling within the ventral tegmental 
area regulates cellular and behavioral responses to stressful stimuli. Biol Psychiatry 64: 691-700.

Lai WS, Xu B, Westphal KG, Paterlini M, Olivier B, Pavlidis P et al (2006). Akt1 deficiency affects neuronal morphology and predisposes to abnormalities in prefrontal cortex functioning. Proc Natl Acad Sci USA 103: 16906-16911.

Le Roy V, Delmas Y, Verdoux H (2009). Chronic renal complications induced by lithium. Encephale 35: 605-610.

Leucht S, Kissling W, McGrath J (2004). Lithium for schizophrenia revisited: a systematic review and meta-analysis of randomized controlled trials. J Clin Psychiatry 65: 177-186.

Mavrikaki M, Nomikos GG, Panagis G (2010). Efficacy of the atypical antipsychotic aripiprazole in d-amphetamine-based preclinical models of mania. Int J Neuropsychopharmacol 13: 541-548.

McGinty JF, Shi XD, Schwendt M, Saylor A, Toda S (2008). Regulation of psychostimulant-induced signaling and gene expression in the striatum. J Neurochem 104: 1440-1449.

O'Brien WT, Harper AD, Jove F, Woodgett JR, Maretto S, Piccolo S et al (2004). Glycogen synthase kinase-3beta haploinsufficiency mimics the behavioral and molecular effects of lithium. J Neurosci 24: 6791-6798.

O'Brien WT, Klein PS (2009). Validating GSK3 as an in vivo target of lithium action. Biochem Soc Trans 37: 1133-1138.

O'Donnell KC, Gould TD (2007). The behavioral actions of lithium in rodent models: leads to develop novel therapeutics. Neurosci Biobehav Rev 31: 932-962.

Perry W, Minassian A, Paulus MP, Young JW, Kincaid MJ, Ferguson EJ et al (2009). A reverse-translational study of dysfunctional exploration in psychiatric disorders: from mice to men. Arch Gen Psychiatry 66: 1072-1080.

Polter A, Beurel E, Yang S, Garner R, Song L, Miller CA et al (2010). Deficiency in the inhibitory serine-phosphorylation of glycogen synthase kinase-3 Increases sensitivity to mood disturbances. Neuropsychopharmacology 35: 1761-1774.

Prickaerts J, Moechars D, Cryns K, Lenaerts I, van Craenendonck $\mathrm{H}$, Goris I et al (2006). Transgenic mice overexpressing glycogen synthase kinase 3beta: a putative model of hyperactivity and mania. J Neurosci 26: 9022-9029.

Ring DB, Johnson KW, Henriksen EJ, Nuss JM, Goff D, Kinnick TR et al (2003). Selective glycogen synthase kinase 3 inhibitors potentiate insulin activation of glucose transport and utilization in vitro and in vivo. Diabetes 52: 588-595.

Rothman RB, Baumann MH (2006). Balance between dopamine and serotonin release modulates behavioral effects of amphetamine-type drugs. Ann NY Acad Sci 1074: 245-260.

Tan HY, Nicodemus KK, Chen Q, Li Z, Brooke JK, Honea R et al (2008). Genetic variation in AKT1 is linked to dopamineassociated prefrontal cortical structure and function in humans. J Clin Invest 118: 2200-2208.

Thiselton DL, Vladimirov VI, Kuo PH, McClay J, Wormley B, Fanous A et al (2008). AKT1 is associated with schizophrenia across multiple symptom dimensions in the Irish study of high density schizophrenia families. Biol Psychiatry 63: 449-457.

Tondo L, Baldessarini RJ (2009). Long-term lithium treatment in the prevention of suicidal behavior in bipolar disorder patients. Epidemiol Psychiatry Soc 18: 179-183.

Vasdev N, Garcia A, Stableford WT, Young AB, Meyer JH, Houle S et al (2005). Synthesis and ex vivo evaluation of carbon-11 labelled $\mathrm{N}$-(4-methoxybenzyl)-N' ${ }^{\prime}$-(5-nitro-1,3-thiazol-2-yl)urea ([11C]AR-A014418): a radiolabelled glycogen synthase kinase3beta specific inhibitor for PET studies. Bioorg Med Chem Lett 15: 5270-5273.

Yuan H, Mao J, Li L, Wu D (1999). Suppression of glycogen synthase kinase activity is not sufficient for leukemia enhancer factor-1 activation. J Biol Chem 274: 30419-30423.

Zhao Z, Robinson RG, Barnett SF, Defeo-Jones D, Jones RE, Hartman GD et al (2008). Development of potent, allosteric dual Akt1 and Akt2 inhibitors with improved physical properties and cell activity. Bioorg Med Chem Lett 18: 49-53.

Zhuang X, Oosting RS, Jones SR, Gainetdinov RR, Miller GW, Caron MG et al (2001). Hyperactivity and impaired response habituation in hyperdopaminergic mice. Proc Natl Acad Sci USA 98: 1982-1987.

Supplementary Information accompanies the paper on the Neuropsychopharmacology website (http://www.nature.com/npp) 